\title{
Synthesis and biological evaluation of new quinoxaline derivatives as antioxidant and anti-inflammatory agents
}

\author{
Asunción Burguete ${ }^{\mathrm{a}, \mathrm{b} 1}$, Eleni Pontiki ${ }^{\mathrm{a}}$, Dimitra Hadjipavlou-Litina, ${ }^{\mathrm{a}^{*}}$ Saioa Ancizu ${ }^{\mathrm{b}}$, \\ Raquel Villar $^{b}$, Beatriz Solano ${ }^{b}$, Elsa Moreno ${ }^{b}$, Enrique Torres ${ }^{b}$, Silvia Pérez ${ }^{b}$, Ignacio \\ Aldana $^{\mathrm{b}}$, Antonio Monge ${ }^{\mathrm{b}}$.
}

We report the synthesis, anti-inflammatory and antioxidant activities of novel quinoxaline and quinoxaline 1,4-di- $N$-oxide derivatives. Microwave assisted methods have been used in order to optimize reaction times and to improve the yields. The tested compounds presented important scavenging activities and promising in vitro inhibition of soybean lipoxygenase. Two of the best lipoxygenase inhibitors (compounds $\mathbf{7 b}$ and $\mathbf{8 f}$ ) were evaluated as in vivo antiinflammatory agents using the carrageenin-induced edema model. One of them (compound $\mathbf{7 b}$ ) showed important in vivo anti-inflammatory effect (41\%) similar to that of indomethacin (47\%) used as the reference drug.

\section{Introduction}

Arachidonic acid (AA) metabolism, mediated by the LOX enzyme family, leads to the generation of leukotrienes, a type of pro-inflammatory mediators, that are involved in processes such as fever, asthma ${ }^{1}$ or cardiovascular disease. ${ }^{2}$ Aberrant AA metabolism is also related to carcinogenesis. In that respect, recent studies revealed increased LOX expression levels in a wide range of cancers including pancreatic, bladder or breast cancer. ${ }^{3-5}$

On the other hand, during the inflammation process, phagocytic leukocytes (e.g. neutrophils, monocytes, macrophages, eosinophils) produce reactive oxygen species (ROS), such as superoxide radical anion, hydrogen peroxide and hydroxyl radical. The presence of high levels of ROS induce and heighten certain pathological conditions such as carcinogenesis, atherosclerosis and neurodegenerative diseases ${ }^{6-8}$ and are well known to be involved in the induction and prolongation of inflammatory process. ${ }^{9,10}$ The involvement of ROS in inflammation is confirmed by a number of commercially available non-steroidal anti-inflammatory drugs NSAIDs (acetaminophen, salicylates, indomethacin and nimesulide) that have been demonstrated to possess radical scavenging properties. ${ }^{11-14}$

Taking into account the above mentioned relationship between ROS and inflammation as well as the importance of inhibition of LOX to combat inflammatory and carcinogenic processes, the development of new compounds having both anti-inflammatory and antioxidant activities and being LOX inhibitors constitutes an interesting approach in the obtention of new drugs for cancer prevention, treatment of chronic inflammation and other related pathological conditions.

Quinoxaline 1,4-di- $N$-oxide derivatives are a class of compounds having a great interest in medicinal chemistry as they display a broad range of biological properties such as antibacterial, anticancer or antiparasitic. ${ }^{15-17}$ We have demonstrate $^{18}$ that quinoxaline 1,4-di- $N$-oxide derivatives show also very interesting antioxidant and antiinflammatory properties, some of them displaying an in vivo anti-inflammatory effect higher than the reference drug, indomethacin IMA, and promising in vitro inhibition values of $\operatorname{LOX}(<1 \mu \mathrm{M})$.

Based on these results and with the aim of obtaining new compounds with improved activities we now describe the synthesis, antioxidant and in vivo anti-inflammatory activity and LOX inhibition of a wide number of new quinoxaline and quinoxaline 1,4-di- $N$-oxide derivatives. Several structural modifications have been carried out (Figure 1) in order to determine the structural requirements of this kind of compounds to act as anti-inflammatory and antioxidant agents.

\section{Results and Discussion}

Synthesis

The new compounds of series 1, 2 and $\mathbf{3}$ were synthesized following the previous described procedures. ${ }^{18}$ The starting reagents, 5-substituted or 5,6-disubstituted benzofuroxanes (BFX) I were obtained by previously reported methods. ${ }^{19}$

\footnotetext{
a, ${ }^{*}$ Department of Pharmaceutical Chemistry, School of Pharmacy, Aristotle University of Thessaloniki, Thessaloniki, 54124, Greece. Email: hadjipav@pharm.auth.gr. Fax:+302310997679. Tel: +302310997627.

${ }^{\mathrm{b}}$ Unidad de Investigación y Desarrollo de Medicamentos, Centro de Investigación en Farmacobiología Aplicada (CIFA), University of Navarra, c/Irunlarrea s/n, 31080 Pamplona, Spain
} 


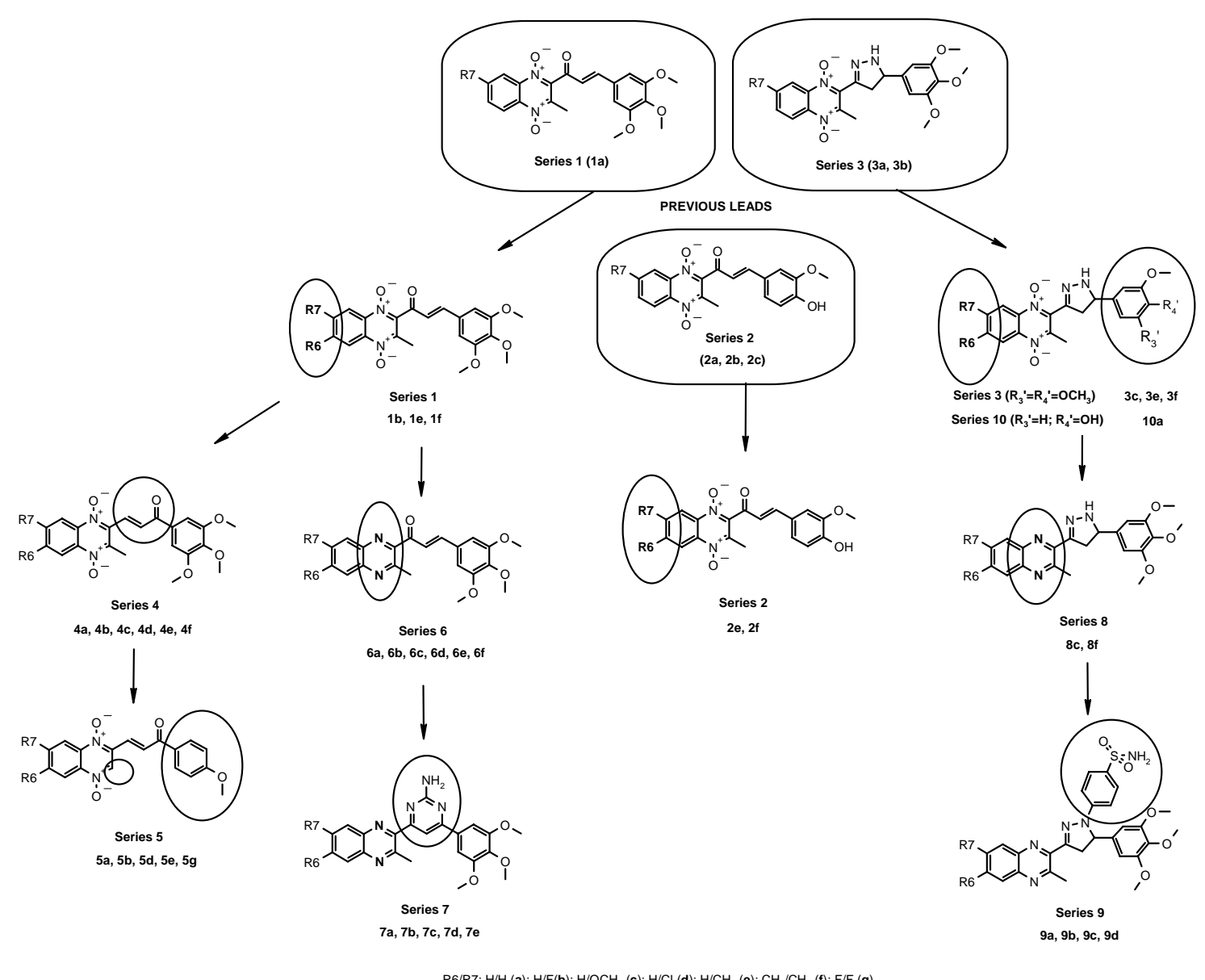

Fig 1. Chemical structure modifications of the previous lead compounds

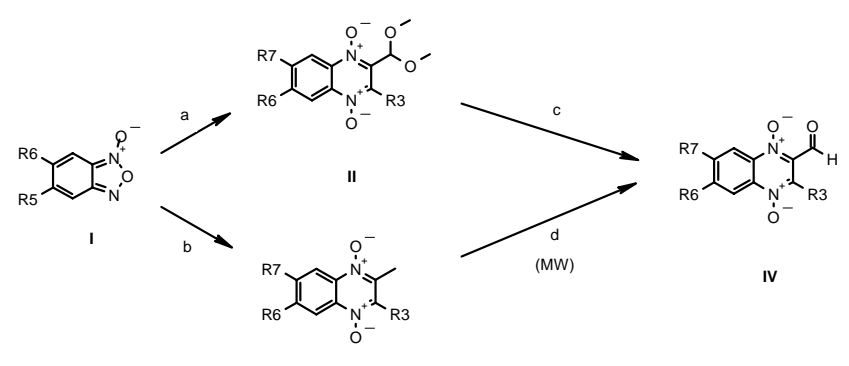

III
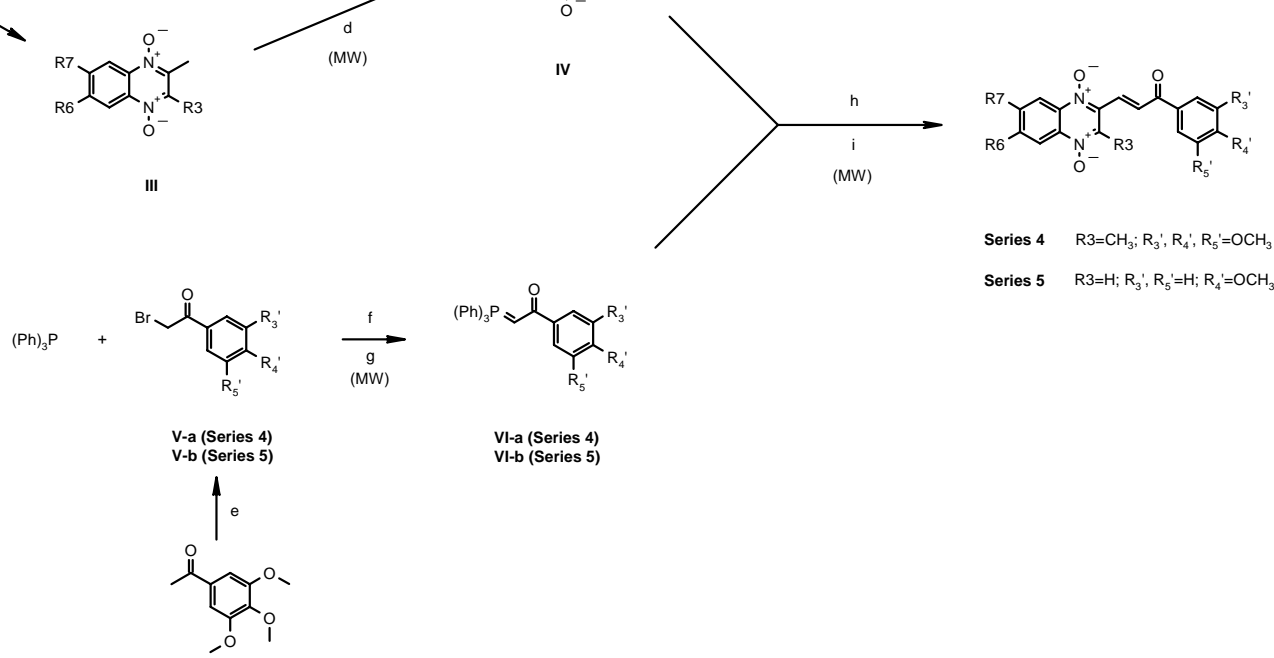

Series $5 \quad R 3=H ; R_{3}^{\prime}, R_{5}^{\prime}=H ; R_{4}^{\prime}=\mathrm{OCH}_{3}$

Scheme 1. Reagents and conditions: (a) 4,4-dimethoxy-butan-2-one (series 4) or 3,3-dimethoxy-propionaldehyde (series 5), pyrrolidine; (b) Butan-2-one (series 4) or Propionaldehyde (series 5), morpholine (c) HCl/MeOH, DCM extraction; (d) $\mathrm{SeO}_{2}$ /acetonitrile, 5', $200 \mathrm{~W}$ (MW); (e) $\mathrm{Br}_{2} / \mathrm{CH}_{3} \mathrm{COOH}$; (f) Toluene, $\mathrm{NaH} / \mathrm{H}_{2} \mathrm{O}$; (g) Xylene, 1'30", 110 W (MW), $\mathrm{NaH} / \mathrm{H}_{2} \mathrm{O}$; (h) DCM reflux, 5/6 hours; (i) $\mathrm{MeOH}, 5$ ', $25 \mathrm{~W}$ (MW) 


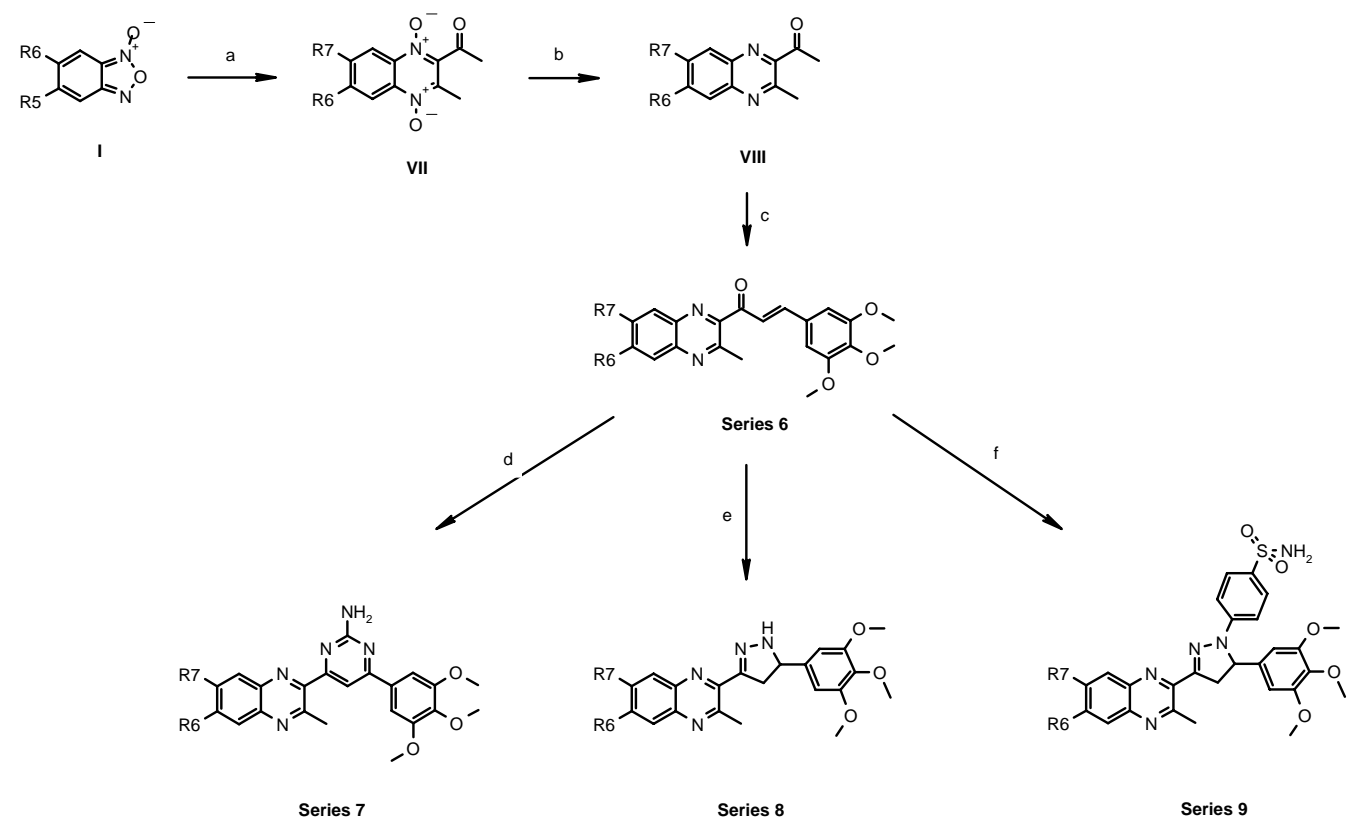

Scheme 2. Reagents and conditions:(a) Pentane-2,4-dione, triethylamine (b) $\mathrm{Na}_{2} \mathrm{~S}_{2} \mathrm{O}_{4}$, methanol, $70^{\circ} \mathrm{C}$; (c) 3,4,5Trimethoxy-benzaldehyde, $3 \% \mathrm{NaOH} /$ methanol, r.t; (d) guanidine hydrochloride, $10 \% \mathrm{KOH} /$ isopropanol reflux, $24 \mathrm{~h}$; (e) $\mathrm{NH}_{2} \mathrm{NH}_{2}$, ethanol, r.t.; (f) 4-hydrazinobenzene-1-sulfonamide hydrochloride 97\%, ethanol, 20', $50 \mathrm{~W}$ (MW)

The classic Claisen-Schmidt condensation, used to synthesize compounds with $\alpha, \beta$-unsaturated ketone system (such as compounds in series 1), did not react in the same expected way to obtain compounds in series $\mathbf{4}$ and $\mathbf{5}$. Thus, a Wittig reaction was proposed to obtain the desired compounds (Scheme 1). Usually, these reactions, that consist in a condensation between an aldehyde and an ylide to afford the corresponding $\alpha, \beta$-unsaturated ketone system derivative, are carried out in a dichloromethane reflux during 5 or 6 hours. ${ }^{20}$ After a deep study of the reaction conditions we optimized a microwave assisted method for the synthesis of these two series concluding that the use of the microwave improved conversion ratios and decreased reaction times. As shown in Scheme 1, both aldehyde and ylide had to be previously synthesized. Aldehydes IV were obtained by two different methods: one of them included deprotection of an acetal group in acid medium; the other involved a methyl oxidation using $\mathrm{SeO}_{2}$ as the oxidant agent. ${ }^{21}$ The second method was found to be the most appropriate for the synthesis of these aldehydes as a huge volume of solvent was needed in the first one to do the extraction. In addition, a microwave assisted method was developed by our group to carry out this reaction. The starting compounds II and III were synthesized by the classic Beirut reaction ${ }^{22}$ between the appropriate BFX and the corresponding carbonilic derivative using pyrrolidine or morpholine as the catalyst. Obtention of ylide VI required a reaction between triphenylphosphine and the corresponding alkyl halide $\mathbf{V}$ and a subsequent treatment with a weak base. ${ }^{20}$ In the case of series $\mathbf{5}$, the halide was commercially available and the formation of the ylide was carried out by using a microwave assisted method. The synthesis of ylide in series $\mathbf{4}$ was performed by a conventional route as the microwave did not work as expected with this reaction. The required halide was previously synthesized as reported ${ }^{20}$ and used without purification to obtain the ylide, that could be the reason of the failure of the microwave assisted synthesis.

Synthesis of series 6 was carried out by a Claisen-Schmidt condensation between the corresponding 6,7substituted 2-acetyl-3-methyl quinoxaline VIII and 3,4,5-Trimethoxy-benzaldehyde using 3\% sodium hydroxide in methanol (Scheme 2). Starting compounds VIII were obtained by reduction of the $N$-oxide groups ${ }^{23}$ of compounds VII $^{24}$ with $\mathrm{Na}_{2} \mathrm{~S}_{2} \mathrm{O}_{4}$. While the reaction to obtain series 1 was performed at low temperature ${ }^{18}$ it was possible to carry out the condensation at room temperature to obtain compounds of series $\mathbf{6}$ and this fact resulted in much better yields.

Cyclization of the $\alpha, \beta$-unsaturated ketone system in compounds of series $\mathbf{6}$ yield compounds of series 7, 8 and 9 (Scheme 2). Formation of a six-membered ring (series 7) was carried out in an isopropanol reflux in the presence of guanidine and $\mathrm{KOH}$ as the catalyst. Reaction between compounds of series $\mathbf{6}$ and hydrazine gave compounds of series $\mathbf{8}$ and in the same way, compounds of series $\mathbf{2}$ reacted with hydrazine to afford compounds of series $\mathbf{1 0}$. The conventional method to synthesize 3,5-substituted 1-(4-sulfamylphenyl) pyrazolines (compounds 9) consists of an eight-hour ethanol reflux. ${ }^{25}$ We optimized a microwave assisted method for the preparation of this series $\mathbf{9}$ and we managed to reduce the volume of solvent, the reaction times and, as a consequence, possible secondary reactions.

\section{Antioxidant activity}

The estimation of the antioxidant potential of the synthesized compounds was assessed by several different assays in order to study a wider spectrum of scavenging properties. The results obtained were compared to well known 
Table 1. Interaction percentage with DPPH (DPPH \%) at $0.05 \mathrm{mM}, 0.1 \mathrm{mM}$ and $0.2 \mathrm{mM}$; Antioxidant determination using the ABTS cation radical-percentage inhibition (ABTS \%)

\begin{tabular}{|c|c|c|c|c|c|c|c|}
\hline \multirow[t]{2}{*}{ Compound } & \multicolumn{3}{|c|}{ DРPH \% } & $\%$ & & & \multirow[t]{2}{*}{$\begin{array}{r}\text { ABTS \% } \\
0.1 \mathrm{mM}\end{array}$} \\
\hline & $20 \mathrm{~min}$ & $60 \mathrm{~min}$ & $20 \mathrm{~min}$ & $60 \mathrm{~min}$ & $20 \mathrm{~min}$ & $60 \mathrm{~min}$ & \\
\hline $1 \mathrm{~b}$ & No & No & 1.4 & 2.3 & 0.4 & 1.7 & 19.2 \\
\hline 1e & 6.5 & 0.8 & 2.5 & 4.3 & No & No & 2.1 \\
\hline 1f & 2.4 & No & No & No & No & No & 8.8 \\
\hline $2 e$ & 11.4 & 12.0 & 28.7 & 37.7 & 39.7 & 50.1 & 92.3 \\
\hline $2 f$ & 15.9 & 18.8 & 32.2 & 41.4 & 50.8 & 62.9 & 84.3 \\
\hline $3 c$ & 58.5 & 65.9 & 58.5 & 70.1 & 65.5 & 78.7 & 48.3 \\
\hline $3 e$ & 44.1 & 54.9 & 40.6 & 55.5 & 55.7 & 66.8 & 40.2 \\
\hline $3 f$ & 24.9 & 41.3 & 53.3 & 62.5 & 89.6 & 94.0 & 50.5 \\
\hline $4 a$ & No & No & 7.5 & 11.9 & 2.8 & No & No \\
\hline $4 b$ & 7.4 & 6.9 & No & 3.3 & No & No & No \\
\hline $4 c$ & 3.2 & 3.9 & 3.9 & 6.0 & No & No & 11.1 \\
\hline $4 d$ & 0.7 & No & 2.4 & No & No & No & No \\
\hline $4 e$ & No & No & 0.6 & 2.9 & 1.0 & 3.6 & 9.1 \\
\hline 4f & 0.3 & No & 2.9 & 1.6 & No & 2.4 & No \\
\hline $5 a$ & 0.6 & 1.1 & 0.8 & 3.0 & 4.4 & No & 12.1 \\
\hline $5 \mathbf{b}$ & 3.7 & 4.5 & 2.1 & No & No & 1.1 & No \\
\hline $5 d$ & 3.3 & 1.8 & No & 2.5 & No & No & 10.0 \\
\hline $5 e$ & No & No & No & 4.0 & No & 0.1 & No \\
\hline $5 g$ & No & No & No & No & No & No & 6.5 \\
\hline $6 a$ & 6.0 & 4.0 & 1.0 & 1.0 & No & No & 39.3 \\
\hline $6 b$ & 2.0 & 4.0 & 2.0 & 2.0 & 1.0 & 2.0 & 81.0 \\
\hline 6c & 7.0 & 3.0 & 3.0 & No & 2.0 & 4.0 & 80.6 \\
\hline 6d & 5.0 & 6.0 & 7.0 & 2.0 & 3.0 & 5.0 & 79.7 \\
\hline $6 e$ & 8.0 & 3.0 & No & No & 5.0 & 3.0 & 72.0 \\
\hline $6 f$ & 3.0 & 3.0 & 6.0 & 1.0 & 5.0 & 5.0 & 55.2 \\
\hline $7 a$ & 1.7 & 7.3 & 4.3 & 0.3 & 5.5 & 3.9 & 18.1 \\
\hline $7 b$ & 6.3 & 8.9 & 10.7 & 14.6 & 13.0 & 18.8 & 40.6 \\
\hline 7c & No & No & No & 0.6 & No & No & No \\
\hline 7d & 0.6 & 1.7 & No & No & No & 0.9 & No \\
\hline $7 e$ & 0.4 & 2.9 & No & 1.3 & No & 2.2 & 4.3 \\
\hline $8 c$ & 11.7 & 22.5 & 19.2 & 37.3 & 26.4 & 45.0 & 74.2 \\
\hline $8 f$ & 13.4 & 23.5 & 24.6 & 42.9 & 35.8 & 52.5 & 76.1 \\
\hline $9 a$ & 5.1 & 4.4 & 6.7 & 8.0 & 10.8 & 6.4 & 2.6 \\
\hline $9 b$ & No & No & No & No & No & No & No \\
\hline 9c & No & No & No & No & No & No & 10.1 \\
\hline 9d & No & No & No & No & No & 1.0 & 5.8 \\
\hline $10 a$ & 49.2 & 60.6 & 62.7 & 72.4 & 83.3 & 87.7 & 71.6 \\
\hline $\begin{array}{l}\text { NDGA } \\
\text { Trolox }\end{array}$ & 68 & 72 & 81 & 83 & & & 88 \\
\hline
\end{tabular}

No: no result under the experimental conditions; each experiment was performed at least in triplicate and the standard deviation of absorbance was less than $10 \%$ of the mean; NDGA nordihydroguaiaretic acid

antioxidants agents such as nordihydroguaeretic acid (NDGA), trolox, and caffeic acid. Most of the experimental procedures required a spectrophotometric measurement and a certain reaction time to obtain reproducible results.

DPPH interaction. One such method that is currently popular is based upon the use of 1,1-diphenyl-2-picrylhydrazyl (DPPH). DPPH is a stable free radical with a spare electron, which is delocalized over the whole molecule. The delocalization causes deep violet color with $\lambda$ max around $517 \mathrm{~nm}$. When an ethanolic solution of DPPH is mixed with that of a compound that can donate a hydrogen atom, then this gives rise to the reduced form with the loss of the characteristic color. This interaction indicates radical scavenging ability in an iron-free system. Our compounds were examined for their DPPH interaction at $0.05 \mathrm{mM}, 0.1 \mathrm{mM}$ and $0.2 \mathrm{mM}$ after 20 and $60 \mathrm{~min}$ (table 1).

Compounds of series 2, 3, 8 and 10 (2e, 2f, 3c, 3e, 3f, 8c, 8f and 10a), showed the best DPPH interaction percentage values, some of them displaying similar values (70-72\%) to that of the reference compound NDGA (83\%) at the same concentration. These compounds have either a phenolic group or a free amino pyrazoline ring in their structure, so they are able to donate a hydrogen atom. The most interesting derivatives were those with the pyrazoline 
moiety (compounds 3c, 3e, 3f, 8c, 8f and 10a) and among them, those with $N$-oxide groups in the quinoxaline ring (3c, 3e, 3f and 10a) exhibited significantly increased activity compared to their reduced analogues (8c and $\mathbf{8 f})$. Thus, the presence of the $N$-oxide groups in these compounds might increase the acidity of the amino group making easier the release of the hydrogen atom and so increasing their scavenging activity. The presence of both $\mathrm{NH}$ and $\mathrm{OH}$ groups in the same molecule (compound 10a) seemed to strengthen its antioxidant character.

For compounds $2 \mathbf{e}, \mathbf{2 f}, 3 \mathbf{c}, 3 \mathbf{e}, \mathbf{3 f}$ the interaction values were found to be time and concentration dependent.

Table 2. Superoxide radical scavenging activity (PMS \%); Competition percentage with DMSO for hydroxyl radical (OH \%); Inhibition of linoleic acid lipid peroxidation induced by AAPH (AAPH)

\begin{tabular}{|c|c|c|c|c|c|}
\hline \multirow{2}{*}{ Compound } & \multicolumn{2}{|c|}{ PMS \% } & \multirow{2}{*}{$\begin{array}{l}\underline{\mathbf{O H} \%} \\
0.1 \mathrm{mM}\end{array}$} & \multicolumn{2}{|c|}{$\underline{\text { AAPH }}$} \\
\hline & $0.01 \mathrm{mM}$ & $0.1 \mathrm{mM}$ & & $\begin{array}{l}\text { AAPH \% } \\
0.1 \mathrm{mM}\end{array}$ & $\mathrm{IC}_{50}(\mathrm{mM})$ \\
\hline $1 b$ & 20.0 & 75.0 & 99.7 & & 0.091 \\
\hline $1 e$ & - & No & 92.5 & & 0.100 \\
\hline 1f & - & No & 91.5 & 35.3 & - \\
\hline $2 e$ & No & 62.5 & No & 24.3 & - \\
\hline $2 f$ & - & 6.3 & 95.9 & 28.2 & - \\
\hline $3 c$ & - & No & 94.4 & & 0.084 \\
\hline $3 \mathbf{e}$ & - & No & 96.0 & 29.0 & - \\
\hline $3 f$ & - & No & 99.6 & 43.8 & - \\
\hline $4 a$ & - & No & 94.5 & No & - \\
\hline $4 b$ & - & 25.0 & 96.3 & No & - \\
\hline $4 c$ & - & No & 97.8 & No & - \\
\hline 4d & - & No & 87.6 & 3.6 & - \\
\hline $4 e$ & - & No & 94.6 & 13.0 & - \\
\hline $4 f$ & - & 57.1 & 98.5 & 18.2 & - \\
\hline $5 a$ & - & 50.0 & 98.4 & 11.2 & - \\
\hline $5 b$ & 50.0 & 96.4 & 96.6 & 6.8 & - \\
\hline $5 d$ & - & No & 95.2 & 10.1 & - \\
\hline $5 e$ & - & No & 97.6 & 22.6 & - \\
\hline $5 g$ & - & 50.0 & 96.9 & 9.0 & - \\
\hline $6 a$ & 81.1 & 79.0 & 91.0 & 50.5 & - \\
\hline $6 b$ & 51.4 & 100 & 87.0 & & 0.010 \\
\hline $6 c$ & 52.8 & 100 & 84.0 & 45.0 & - \\
\hline 6d & 52.8 & 100 & 85.0 & 21.5 & - \\
\hline $6 e$ & 97.2 & 100 & 85.0 & & $<0.010$ \\
\hline $6 f$ & 81.1 & 78.9 & 92.0 & 45.4 & - \\
\hline $7 a$ & - & No & No & & 0.053 \\
\hline $7 \mathbf{b}$ & - & 50.0 & No & & 0.073 \\
\hline 7c & - & No & 77.7 & & 0.094 \\
\hline $7 d$ & - & 31.3 & 91.8 & & 0.078 \\
\hline $7 e$ & - & No & No & & 0.089 \\
\hline $8 c$ & - & No & No & 2.9 & - \\
\hline $8 f$ & - & No & 82.6 & & 0.078 \\
\hline 9a & - & No & 92.9 & & 0.023 \\
\hline $9 b$ & - & No & 97.4 & & 0.072 \\
\hline $9 c$ & - & No & 92.6 & 8.5 & - \\
\hline 9d & - & No & 87.0 & & 0.077 \\
\hline $10 a$ & 50.0 & 68.8 & 98.3 & & 0.074 \\
\hline $\begin{array}{c}\text { Caffeic acid } \\
\text { Ascorbic acid } \\
\text { Trolox }\end{array}$ & 45 & & 88.2 & $\begin{array}{l}95.8 \\
63.0\end{array}$ & \\
\hline
\end{tabular}

No: no result under the experimental conditions; - not tested; each experiment was performed at least in triplicate and the standard deviation of absorbance was less than $10 \%$ of the mean

ABTS assay. Another existing method to evaluate antioxidant activity is the ABTS radical cation decolorization assay. ${ }^{26}$ The pre-formed radical monocation of 2,2'-azinobis-(3-ethylbenzothiazoline-6-sulfonic acid) (ABTS ${ }^{+}$) is generated by oxidation of ABTS with potassium persulfate and reduced in the presence of hydrogen-donating 
antioxidants. The decolorization of the blue/green solution (due to the $\mathrm{ABTS}^{+}$color) after the treatment with the compound is indicative of its scavenging ability.

In the same way as DPPH interaction, the best activities in the ABTS assay (table 1) were shown by compounds of series 2, 3, 8 and $10(2 e, 2 f, 3 c, 3 e, 3 f, 8 c, 8 f$ and 10a) with a phenolic group and/or a free amino pyrazoline ring in the structure. Nevertheless, these activities seemed to have the opposite trend of that showed in the DPPH assay, as the best derivatives were those with a phenol group (compounds $2 \mathbf{e}$ and $\mathbf{2 f}$ ) followed by compounds of series 8 (8c and $\mathbf{8 f}$ ) and series $\mathbf{3}$ (3c, 3e and 3f). Surprisingly, compounds of series 6 (6a-f)showed quite good values of ABTS ${ }^{+}$ scavenging ability as well as compound $\mathbf{7 b}$ that displayed an activity similar to that of compounds of series $\mathbf{3}$.

Superoxide radical anion $\left(\cdot \mathrm{O}_{2}^{-}\right)$and hydroxyl radical $(\cdot \mathbf{O H})$ scavenging activity. The ability of the compounds to scavenge superoxide radical anion and hydroxyl radical was also evaluated. Superoxide anion is considered to be the "primary" ROS that initiates the generation of other ROS such as hydrogen peroxide and hydroxyl radicals. ${ }^{27}$ These toxic oxygen species are involved in the oxidative damage to DNA, proteins and other macromolecules leading to the development of several degenerative diseases associated with aging. ${ }^{28}$

Generation of non enzymatic superoxide anion radicals was carried out by mixing phenazine methosulfate (PMS), nicotinamide adenine dinucleotide NADH and air-oxygen. The production of superoxide was estimated by the nitroblue tetrazolium method. ${ }^{29}$ As shown in the table 2 compounds with an $\alpha, \beta$-unsaturated ketone system $(\mathbf{1 b}, \mathbf{2 e}$, 4f, 5a, 5b, 5g and 6a-f) presented the best superoxide scavenging activities (higher than that of Caffeic acid used as the reference compound) being the reduced derivatives (6a-f) the most interesting structures with interaction values between 79 and $100 \%$. This evidence led us to affirm that the olefinic moiety might play an important role in the activity of these compounds by trapping the superoxide radical. The presence of the $N$-oxide groups in the molecules greatly decreases their scavenging ability.

The competition of compounds with dimethyl sulfoxide (DMSO) for $\cdot \mathrm{OH}$ radicals, generated by the $\mathrm{Fe}^{3+} /$ ascorbic $^{2}$ acid system, expressed as the inhibition of formaldehyde production, was used for the evaluation of their hydroxyl radical scavenging activity. Most of the tested compounds exhibited high competition percentage values at $0.1 \mathrm{mM}$ (Table 2), similar to that of Trolox used as the reference compound, which indicates that these derivatives are good hydroxyl radical scavengers.

Inhibition of linoleic acid lipid peroxidation. Lipid peroxidation in cell membrane has been proposed to be a major mechanism for several pathological events including cancer, Parkinson's disease and aging. ${ }^{30}$ The ability of the compounds to inhibit lipid peroxidation was measured. ${ }^{31}$ Production of conjugated diene hydroperoxide by oxidation of linoleic acid in an aqueous dispersion was monitored at $234 \mathrm{~nm}$. 2,2'-Azobis(2-amidinopropane) dihydrochloride (AAPH) was used as a free radical initiator.

The results (Table 2) showed that compounds $\mathbf{6 b}$ and $\mathbf{6 e}$ were the best inhibitors of lipid peroxidation displaying $\mathrm{IC}_{50}$ values of $0.01 \mathrm{mM}$ and $<0.01 \mathrm{mM}$ respectively. Compounds of series $\mathbf{7}$ (7a, 7b, 7d and 7e) and 9 (9b and 9d), having a free amino group in their structure, also presented good inhibition activities, especially those with a hydrogen atom (7a and 9a) in position R7 of the quinoxaline ring. Replacement of the hydrogen by a fluoro atom (7b and $9 \mathrm{~b}$ ) resulted in a decrease in the activity, although fluoro derivatives showed also interesting inhibition values of lipid peroxidation.

\section{Inhibition of Soybean lipoxygenase (LOX)}

The assay for lipoxygenase (LOX) activity was carried out according to the UV absorbance based enzyme assay ${ }^{32}$, ${ }^{33}$ using soybean lipoxygenase. While one may not extrapolate the quantitative results of this assay to the inhibition of mammalian 5-LOX, it has been shown that inhibition of plant LOX activity by NSAIDs is qualitatively similar to their inhibition of the rat mast cell LOX and may be used as a simple qualitative screen for such activity.

In general, compounds that displayed good activities as inhibitors of lipid peroxidation also presented good values of inhibition of LOX (table 3). Thus, the best $\mathrm{IC}_{50}$ values were shown by compounds of series 7 and 9 $\mathbf{( 7 b}>\mathbf{7 e}>\mathbf{9 b}>\mathbf{8 f}>\mathbf{9 d}>\mathbf{7 a})$. From series $\mathbf{8}$ only compound $8 \mathrm{f}$ presented significant activity. Nevertheless, while the best inhibitors of lipid peroxidation were those compounds without any substitution in the quinoxaline ring, the most interesting activities of LOX inhibition were obtained by fluoro and methyl substituted derivatives.

\section{In vivo anti-inflammatory activity}

Two compounds, $\mathbf{7 b}$ (which was the most potent LOX inhibitor) and $\mathbf{8 f}$ (the most potent from series 8), were tested as in vivo anti-inflammatory agents. In acute toxicity experiments, the in vivo examined compounds $\mathbf{7 b}$ and $\mathbf{8 f}$ did not present toxic effects in doses up to $0.2 \mathrm{mmol} / \mathrm{kg}$ body weight. Ulcerogenicity was not found. Acute inflammation is due to the release of chemical mediators, which cause edema as a result of extravasations of fluid and proteins from the local microvasculature and accumulation of polymorphonuclear leukocytes at the inflammatory site. The in vivo anti-inflammarory effects of the tested quinoxaline derivatives were assessed by using the carrageenin-induced rat paw edema (CPE) model and are presented in Table 3 as percentage of weight increase at the right hind paw. The induced edema is a non-specific inflammation highly sensitive to non-steroidal anti-inflammatory agents (NSAIDs). Thus it has been accepted as a useful tool for studying new anti-inflammatory agents. ${ }^{34}$ It reliably 
predicts the anti-inflammatory potency of the NSAIDs and detects during the second phase that are anti-inflammatory agents as a result of inhibition of prostaglandin amplification. ${ }^{35}$ Compound $\mathbf{7 b}$ showed $41.3 \%$ percentage of

Table 3. Inhibition percentage of induced carrageenin rat paw edema (CPE \%) at $0.01 \mathrm{mmol} / \mathrm{kg}$; In vitro inhibition of soybean lipoxygenase (LOX) inhitibion $\%$ at $0.1 \mathrm{mM} / \mathrm{IC}_{50}(\mathrm{mM})$

\begin{tabular}{|c|c|c|c|c|}
\hline \multirow{2}{*}{ Compound } & \multirow{2}{*}{$\underline{\text { CPE \% }}^{\mathbf{a}}$} & \multicolumn{2}{|c|}{$\underline{\text { LOX }}$} & \multirow[b]{2}{*}{$\mathrm{Clog} P^{\mathrm{b}}$} \\
\hline & & $\begin{array}{l}\text { inhibition \% at } \\
0.1 \mathrm{mM}\end{array}$ & $\mathrm{IC}_{50}(\mathrm{mM})$ & \\
\hline $1 b$ & & & 0.090 & -0.01 \\
\hline 1e & & 35.4 & - & 0.20 \\
\hline 1f & & 36.7 & - & 0.64 \\
\hline $2 e$ & & 12.2 & - & 0.08 \\
\hline $2 f$ & & 26.0 & - & 0.53 \\
\hline $3 c$ & & 38.4 & - & -0.33 \\
\hline $3 e$ & & & 0.095 & -0.09 \\
\hline $3 f$ & & & 0.100 & 0.35 \\
\hline $4 a$ & & 30.0 & - & 0.17 \\
\hline $4 b$ & & No & - & 0.46 \\
\hline $4 c$ & & 26.6 & - & 0.43 \\
\hline 4d & & 40.3 & - & 1.03 \\
\hline $4 e$ & & 40.4 & - & 0.67 \\
\hline $4 f$ & & 18.7 & - & 1.12 \\
\hline $5 a$ & & 4.0 & - & 0.29 \\
\hline $5 \mathbf{b}$ & & 19.7 & - & 0.58 \\
\hline $5 d$ & & 2.4 & - & 1.15 \\
\hline $5 e$ & & 5.0 & - & 0.79 \\
\hline $5 g$ & & 23 & - & 0.71 \\
\hline $6 a$ & & & 0.140 & 2.49 \\
\hline $6 b$ & & & 0.200 & 2.60 \\
\hline $6 c$ & & & 0.320 & 2.82 \\
\hline 6d & & & 0.350 & 3.17 \\
\hline $6 e$ & & & 0.088 & 3.37 \\
\hline $6 f$ & & & 0.410 & 2.93 \\
\hline $7 a$ & & & 0.050 & 3.00 \\
\hline $7 b$ & $41.3^{*}$ & & 0.023 & 3.14 \\
\hline $7 c$ & & 21.6 & - & 3.19 \\
\hline $7 d$ & & & 0.089 & 3.71 \\
\hline $7 e$ & & & 0.036 & 3.50 \\
\hline $8 c$ & & 37.9 & - & 2.53 \\
\hline $8 f$ & $28.2 *$ & & 0.042 & 3.08 \\
\hline $9 a$ & & 3.0 & - & 3.90 \\
\hline $9 b$ & & & 0.037 & 4.07 \\
\hline $9 c$ & & No & - & 4.29 \\
\hline 9d & & & 0.043 & 4.64 \\
\hline 10a & & & 0.092 & -0.71 \\
\hline $\begin{array}{c}\text { Caffeic acid } \\
\text { IMA }\end{array}$ & $47 *$ & & 0.600 & \\
\hline
\end{tabular}

No: no result under the experimental conditions; - not tested; each experiment was performed at least in triplicate and the standard deviation of absorbance was less than $10 \%$ of the mean; IMA indomethacin; ${ }^{a}$ statistical studies were done with student's $T$-test, ${ }^{*} p<0.01 ;^{b}$ Reference 37 in the text.

protection while the reference drug indomethacin induced $47 \%$ protection at an equivalent dose. Compound $8 \mathrm{f}$ was less potent (28.2\%). No role for lipophilicity was found. Both derivatives presented similar Clog $P$ values (Table 3).

\section{Experimental Section}

\section{Chemistry}


Microwave assisted synthesis was carried out in a Discover S-Class microwave system apparatus (CEM Corporation). The ${ }^{1} \mathrm{H}$ NMR spectra were recorded on a Bruker 400 Ultrashield $^{\mathrm{TM}}$ (Bruker BioSpin $\mathrm{GmbH}$, Rheinstetten, Germany), using TMS as the internal standard and with $\mathrm{CDCl}_{3}$ and DMSO- $d_{6}$ as the solvents; the chemical shifts are reported in ppm $(\delta)$ and the coupling constant $(J)$ values are given in Hertz $(\mathrm{Hz})$. Signal multiplicities are represented by: s (singlet), bs (broad singlet), d (doublet), dd (double doublet), $\mathrm{t}$ (triplet), and $\mathrm{m}$ (multiplet) The IR spectra were performed on Thermo Nicolet FT-IR Nexus Euro (Madison, USA) using KBr pellets; the frequencies are expressed in $\mathrm{cm}^{-1}$. Elemental microanalyses were obtained on an Elemental Analyzer LECO CHN-900 (Michigan, USA) from vacuum-dried samples. The analytical results for $\mathrm{C}, \mathrm{H}$, and $\mathrm{N}$ were within \pm 0.4 of the theoretical values, indicating a purity of $>95 \%$.

Alugram ${ }^{\circledR}$ SIL G/UV254 (Layer: 0.2 mm) (Macherey-Nagel GmbH \& Co. KG. Postfach 101352. D-52313 Düren, Germany) was used for Thin Layer Chromatography and Silica gel $60(0.040-0.063 \mathrm{~mm})$ for Column Flash Chromatography (Merck).

All reagents and solvents were purchased from commercial sources. E. Merck (Darmstadt, Germany), Scharlau (F.E.R.O.S.A., Barcelona, Spain), Panreac Química S.A. (Montcada i Reixac, Barcelona, Spain), Sigma-Aldrich Química, S.A., (Alcobendas, Madrid), Acros Organics (Janssen Pharmaceuticalaan 3a, 2440 Geel, België) and Lancaster (Bischheim-Strasbourg, France).

General method for the microwave assisted synthesis of 1,4-dioxy-quinoxaline-2-carbaldehyde derivatives (compounds IV). In a microwave reaction flask $3 \mathrm{mmol}$ of the appropriate compound III, $4.5 \mathrm{mmol}$ of $\mathrm{SeO}_{2}$ and 20 $\mathrm{mL}$ of acetonitrile were mixed. The reaction was carried out at $200 \mathrm{~W}$ for 5 minutes. The solvent was evaporated, an extraction with chloroform and water was performed and the residue purified by column chromatography using toluene:dioxane (3:2). The compounds obtained have been previously described. ${ }^{20,36}$

General method for the microwave assisted synthesis of 1-(substituted-phenyl)-2-(triphenyl-phosphonylidene)ethanone derivatives (compounds VI). $5 \mathrm{mmol}$ of triphenylphosphine, $5 \mathrm{mmol}$ of the corresponding halide $\mathbf{V}$ and 3 $\mathrm{mL}$ of xylene were mixed in a microwave reaction flask. The program was run for 1 minute and 30 seconds at $110 \mathrm{~W}$. The residue was dissolved in $10 \mathrm{~mL}$ of methanol and $8 \mathrm{mmol}$ of $\mathrm{NaH}$ in water were added. The mixture was extracted with ethyl ether $(3 \times 25 \mathrm{~mL})$, the organic phase dried with anhydrous $\mathrm{Na}_{2} \mathrm{SO}_{4}$ and the solvent removed under vacuum to yield compounds VI. Spectroscopic data for compounds VI are listed below.

1-(3,4,5-trimethoxy-phenyl)-2-(triphenyl-phosphonylidene)-ethanone (VI-a). The compound VI-a was obtained as a white solid (42\%). IR $(\mathrm{KBr}) 1664(\mathrm{C}=\mathrm{O}), 1584-1463\left(\mathrm{C}-\mathrm{C}\right.$ ar), $1122(\mathrm{C}-\mathrm{O}-\mathrm{C}) ;{ }^{1} \mathrm{NMR}\left(\mathrm{CDCl}_{3}\right) \delta: 3.88(\mathrm{~s}, 3 \mathrm{H}, p-$ $\left.\mathrm{OCH}_{3}\right), 3.92\left(\mathrm{~s}, 6 \mathrm{H}, m-\mathrm{OCH}_{3}\right), 4.35-4.40\left(\mathrm{~d}, 1 \mathrm{H},=\mathrm{CH}, J_{\mathrm{H}-\mathrm{P}}=19.05 \mathrm{~Hz}\right), 7.26\left(\mathrm{~s}, 2 \mathrm{H}, \mathrm{H}_{2}+\mathrm{H}_{6}\right), 7.48-7.53(\mathrm{~m}, 6 \mathrm{H}$, $\left.\mathrm{H}_{3}{ }^{\prime}+\mathrm{H}_{5}{ }^{\prime}\right)$, 7.57-7.61 (m, 3H, $\left.\mathrm{H}_{4}{ }^{\prime}\right)$, 7.71-7.76 (m, 6H, $\left.\mathrm{H}_{2}{ }^{\prime}+\mathrm{H}_{6}{ }^{\prime}\right)$; Analysis calculated for $\mathrm{C}_{29} \mathrm{H}_{27} \mathrm{O}_{4} \mathrm{P}(470.51)$ : C, 74.04; H, 5.74; N, 0.00. Found: C, 73.82; H, 5.70; N, 0.00.

1-(4-methoxy-phenyl)-2-(triphenyl-phosphonylidene)-ethanone (VI-b). The compound VI-b was obtained as a white solid (37\%). IR (KBr) $1649(\mathrm{C}=\mathrm{O}), 1598-1482$ (C-C ar), 1184 (C-O-C); ${ }^{1} \mathrm{NMR}\left(\mathrm{DMSO}-\mathrm{d}_{6}\right) \delta: 3.77(\mathrm{~s}, 3 \mathrm{H}$, $\left.\mathrm{OCH}_{3}\right), 4.37-4.44\left(\mathrm{~d}, 1 \mathrm{H},=\mathrm{CH}, J_{\mathrm{H}-\mathrm{P}}=24.95 \mathrm{~Hz}\right), 6.88-6.90\left(\mathrm{~d}, 2 \mathrm{H}, \mathrm{H}_{3}+\mathrm{H}_{5}, J_{32 / 56}=8.76 \mathrm{~Hz}\right), 7.54-7.59(\mathrm{~m}, 6 \mathrm{H}$, $\left.\mathrm{H}_{3}{ }^{\prime}+\mathrm{H}_{5}{ }^{\prime}\right)$, 7.64-7.70 (m, 6H, $\left.\mathrm{H}_{2}{ }^{\prime}+\mathrm{H}_{4}{ }^{\prime}+\mathrm{H}_{6}{ }^{\prime}\right), 7.81-7.83\left(\mathrm{~d}, 2 \mathrm{H}, \mathrm{H}_{2}+\mathrm{H}_{6}, J_{23 / 65}=8.74 \mathrm{~Hz}\right)$; Analysis calculated for $\mathrm{C}_{27} \mathrm{H}_{23} \mathrm{O}_{2} \mathrm{P}$ (410.46): C, 79.01; H, 5.65; N, 0.00. Found: C, 78.65; H, 5.60; N, 0.00 .

Synthesis of 1-(3-Methyl-quinoxalin-2-yl)-ethanone derivatives (Compounds VIII). These compounds were obtained following the procedure described in the literature. ${ }^{23}$ Spectroscopic data for one of these derivatives (VIIIa, $\mathrm{R} 6 / \mathrm{R} 7=\mathrm{H} / \mathrm{H}$ ) is described below as a reference compound.

1-(3-Methyl-quinoxalin-2-yl)-ethanone (VIIIa). A $218 \mathrm{mg}$ portion of 2-Acetyl-3-methylquinoxaline 1,4-di- $N$-oxide $(1 \mathrm{mmol})$ was dissolved in $20 \mathrm{~mL}$ of methanol. The solution was heated at $70^{\circ} \mathrm{C}$ and $1.04 \mathrm{~g}$ of $\mathrm{Na}_{2} \mathrm{~S}_{2} \mathrm{O}_{4}(6 \mathrm{mmol})$ in $10 \mathrm{~mL}$ of water were added. The mixture was stirred for 10 minutes and the solvent removed under reduced pressure. The solid obtained $\left(50 \%\right.$ yield) was filtered and washed with water. IR $(\mathrm{KBr}) 1694(\mathrm{C}=\mathrm{O})$; ${ }^{1} \mathrm{H}-\mathrm{NMR}\left(\mathrm{DMSO}-\mathrm{d}_{6}\right) \delta$ : $2.76\left(\mathrm{~s}, 3 \mathrm{H}, 3-\mathrm{CH}_{3}\right), 2.84\left(\mathrm{~s}, 3 \mathrm{H}, \mathrm{CO}-\mathrm{CH}_{3}\right), 7.86-7.90\left(\mathrm{ddd}, 1 \mathrm{H}, \mathrm{H}_{6}, J_{65}=8.31 \mathrm{~Hz}, J_{67}=6.93 \mathrm{~Hz}, J_{68}=1.49 \mathrm{~Hz}\right), 7.93-$ $7.97\left(\mathrm{ddd}, 1 \mathrm{H}, \mathrm{H}_{7}, J_{78}=8.38 \mathrm{~Hz}, J_{76}=6.92 \mathrm{~Hz}, J_{75}=1.52 \mathrm{~Hz}\right), 8.05-8.07\left(\mathrm{dd}, 1 \mathrm{H}, \mathrm{H}_{5}, J_{56}=8.34 \mathrm{~Hz}, J_{57}=1.09 \mathrm{~Hz}\right), 8.14-$ $8.16\left(\mathrm{dd}, 1 \mathrm{H}, \mathrm{H}_{8}, J_{87}=8.28 \mathrm{~Hz}, J_{86}=1.08 \mathrm{~Hz}\right.$ ); Analysis calculated for $\mathrm{C}_{11} \mathrm{H}_{10} \mathrm{~N}_{2} \mathrm{O}$ (186.22): $\mathrm{C}, 70.95 ; \mathrm{H}, 5.41 ; \mathrm{N}$, 15.04. Found: $\mathrm{C}, 70.63 ; \mathrm{H}, 5.33 ; \mathrm{N}, 15.08$.

General method for the microwave assisted synthesis of 3-(3-Methyl-1,4-dioxy-quinoxalin-2-yl)-1-(3,4,5trimethoxy-phenyl)-propenone derivatives (Series 4) and of 3-(1,4-dioxy-quinoxalin-2-yl)-1-(4-methoxyphenyl)-propenone derivatives (Series 5). In a microwave reaction flask $0.25 \mathrm{mmol}$ of aldehyde IV, $0.375 \mathrm{mmol}$ of the corresponding ylide VI and $1 \mathrm{~mL}$ of methanol were mixed. The reaction was carried out at $25 \mathrm{~W}$ for 5 minutes. The solid obtained was filtered and purified by flash column chromatography using toluene:dioxane $(3: 2)$ as the eluent.

(2E)-3-(3-Methyl-1,4-dioxy-quinoxalin-2-yl)-1-(3,4,5-trimethoxy-phenyl)-propenone (4a). The derivative 4a was obtained as yellow solid (27\%). IR (KBr) $1651(\mathrm{C}=\mathrm{O}), 1317(\mathrm{~N}-\mathrm{O}), 1120(\mathrm{C}-\mathrm{O}-\mathrm{C}) ;{ }^{1} \mathrm{H}-\mathrm{NMR}\left(\mathrm{CDCl}_{3}\right)$ 8: 2.95 (s, 3H, 
$\left.\mathrm{CH}_{3}\right), 3.98\left(\mathrm{~s}, 3 \mathrm{H}, p-\mathrm{OCH}_{3}\right), 4.00\left(\mathrm{~s}, 6 \mathrm{H}, \mathrm{m}-\mathrm{OCH}_{3}\right), 7.40\left(\mathrm{~s}, 2 \mathrm{H}, \mathrm{H}_{2}{ }^{\prime}+\mathrm{H}_{6}{ }^{\prime}\right), 7.93-7.81\left(\mathrm{~m}, 3 \mathrm{H}, \mathrm{H}_{6}+\mathrm{H}_{7}+\mathrm{H}_{\mathrm{b}}\right), 8.73-8.62$ $\left(\mathrm{m}, 2 \mathrm{H}, \mathrm{H}_{5}+\mathrm{H}_{8}\right), 9.20\left(\mathrm{~d}, 1 \mathrm{H}, \mathrm{H}_{\mathrm{a}}, \mathrm{J}_{\mathrm{ab}}=15.26 \mathrm{~Hz}\right)$; Analysis calculated for $\mathrm{C}_{21} \mathrm{H}_{20} \mathrm{~N}_{2} \mathrm{O}_{6}(396.40): \mathrm{C}, 63.60 ; \mathrm{H}, 5.09$; N, 7.07. Found: C, $63.73 ; \mathrm{H}, 4.99 ; \mathrm{N}, 6.90$.

(2E)-3-(7-Fluoro-3-methyl-1,4-dioxy-quinoxalin-2-yl)-1-(3,4,5-trimethoxy-phenyl)-propenone (4b). The compound $4 \mathbf{b}$ was obtained as yellow solid $(24 \%)$. IR (KBr) $1650(\mathrm{C}=\mathrm{O}), 1319(\mathrm{~N}-\mathrm{O}), 1120(\mathrm{C}-\mathrm{O}-\mathrm{C}) ;{ }^{1} \mathrm{H}-\mathrm{NMR}$ $\left(\mathrm{CDCl}_{3}\right) \delta: 2.93\left(\mathrm{~s}, 3 \mathrm{H}, \mathrm{CH}_{3}\right), 3.99\left(\mathrm{~s}, 3 \mathrm{H}, p-\mathrm{OCH}_{3}\right), 4.00\left(\mathrm{~s}, 6 \mathrm{H}, m-\mathrm{OCH}_{3}\right), 7.40\left(\mathrm{~s}, 2 \mathrm{H}, \mathrm{H}_{2}{ }^{\prime}+\mathrm{H}_{6}{ }^{\prime}\right), 7.61-7.65$ (ddd, $\left.1 \mathrm{H}, \mathrm{H}_{6}, J_{65}=9.61 \mathrm{~Hz}, J_{6 \mathrm{~F}}=7.35 \mathrm{~Hz}, J_{68}=2.46 \mathrm{~Hz}\right), 7.82-7.86\left(\mathrm{~d}, 1 \mathrm{H}, \mathrm{H}_{\mathrm{b}}, J_{\mathrm{ba}}=15.24 \mathrm{~Hz}\right), 8.31-8.34\left(\mathrm{dd}, 1 \mathrm{H}, \mathrm{H}_{8}, \mathrm{~J}_{8 \mathrm{~F}}=\right.$ $\left.8.67 \mathrm{~Hz}, \mathrm{~J}_{86}=2.59 \mathrm{~Hz}\right), 8.70-8.74\left(\mathrm{dd}, 1 \mathrm{H}, \mathrm{H}_{5}, J_{56}=9.48 \mathrm{~Hz}, J_{5 \mathrm{~F}}=5.09 \mathrm{~Hz}\right), 9.20-9.23\left(\mathrm{~d}, 1 \mathrm{H}, \mathrm{H}_{\mathrm{a}}, J_{\mathrm{ab}}=15.25 \mathrm{~Hz}\right)$; Analysis calculated for $\mathrm{C}_{21} \mathrm{H}_{19} \mathrm{FN}_{2} \mathrm{O}_{6}$ (414.38): C, 60.87; H, 4.62; N, 6.76. Found: C, 60.62; H, 4.76; N, 6.51.

(2E)-3-(7-Methoxy-3-methyl-1,4-dioxy-quinoxalin-2-yl)-1-(3,4,5-trimethoxy-phenyl)-propenone (4c). The derivative 4c was obtained as yellow solid (37\%). IR (KBr) $1657(\mathrm{C}=\mathrm{O}), 1320(\mathrm{~N}-\mathrm{O}), 1133$ (C-O-C); ${ }^{1} \mathrm{H}-\mathrm{NMR}$ $\left(\mathrm{CDCl}_{3}\right) \delta: 2.90\left(\mathrm{~s}, 3 \mathrm{H}, \mathrm{CH}_{3}\right), 3.97\left(\mathrm{~s}, 3 \mathrm{H}, p-\mathrm{OCH}_{3}\right), 3.99\left(\mathrm{~s}, 6 \mathrm{H}, m-\mathrm{OCH}_{3}\right), 4.05(\mathrm{~s}, 3 \mathrm{H}$, quinox-OCH$), 7.39(\mathrm{~s}, 2 \mathrm{H}$, $\left.\mathrm{H}_{2}{ }^{\prime}+\mathrm{H}_{6}{ }^{\prime}\right), 7.44-7.47$ (dd, $\left.1 \mathrm{H}, \mathrm{H}_{6}, J_{65}=9.43 \mathrm{~Hz}, J_{68}=2.61 \mathrm{~Hz}\right), 7.84-7.87\left(\mathrm{~d}, 1 \mathrm{H}, \mathrm{H}_{\mathrm{b}}, J_{\mathrm{ba}}=15.23 \mathrm{~Hz}\right), 7.94\left(\mathrm{~d}, 1 \mathrm{H}, \mathrm{H}_{8}\right.$, $\left.J_{86}=2.68 \mathrm{~Hz}\right), 8.55-8.58\left(\mathrm{~d}, 1 \mathrm{H}, \mathrm{H}_{5}, J_{56}=9.45 \mathrm{~Hz}\right), 9.13-9.17\left(\mathrm{~d}, 1 \mathrm{H}, \mathrm{H}_{\mathrm{a}}, J_{\mathrm{ab}}=15.28 \mathrm{~Hz}\right)$; Analysis calculated for $\mathrm{C}_{22} \mathrm{H}_{22} \mathrm{~N}_{2} \mathrm{O}_{7}$ (426.43): C, 61.97; H, 5.20; N, 6.57. Found: C, 61.63; H, 5.66; N, 6.14.

(2E)-3-(7-Chloro-3-methyl-1,4-dioxy-quinoxalin-2-yl)-1-(3-hydroxy-4,5-dimethoxy-phenyl)-propenone (4d). The derivative 4d was obtained as orange solid (30\%). IR (KBr) 1654 (C=O), 1321 (N-O), 1135 (C-O-C); ${ }^{1} \mathrm{H}-\mathrm{NMR}$ $\left(\mathrm{CDCl}_{3}\right) \delta: 2.93\left(\mathrm{~s}, 3 \mathrm{H}, \mathrm{CH}_{3}\right), 3.99\left(\mathrm{~s}, 3 \mathrm{H}, p-\mathrm{OCH}_{3}\right), 4.00\left(\mathrm{~s}, 6 \mathrm{H}, m-\mathrm{OCH}_{3}\right), 7.39\left(\mathrm{~s}, 2 \mathrm{H}, \mathrm{H}_{2}{ }^{\prime}+\mathrm{H}_{6}{ }^{\prime}\right), 7.81-7.84(\mathrm{dd}, 1 \mathrm{H}$, $\left.\mathrm{H}_{6}, J_{65}=9.11 \mathrm{~Hz}, J_{68}=2.18 \mathrm{~Hz}\right), 7.81-7.85\left(\mathrm{~d}, 1 \mathrm{H}, \mathrm{H}_{\mathrm{b}}, J_{\mathrm{ba}}=15.20 \mathrm{~Hz}\right), 8.63-8.65\left(\mathrm{~d}, 1 \mathrm{H}, \mathrm{H}_{5}, J_{56}=9.13 \mathrm{~Hz}\right), 8.66-8.67(\mathrm{~d}$, $\left.1 \mathrm{H}, \mathrm{H}_{8}, J_{86}=2.15 \mathrm{~Hz}\right), 8.19-8.23\left(\mathrm{~d}, 1 \mathrm{H}, \mathrm{H}_{\mathrm{a}}, J_{\mathrm{ab}}=15.25 \mathrm{~Hz}\right)$; Analysis calculated for $\mathrm{C}_{21} \mathrm{H}_{19} \mathrm{ClN}_{2} \mathrm{O}_{6}(430.89)$ : C, 58.54; H, 4.45; N, 6.50. Found: C, 58.58; H, 4.41; N, 6.32.

(2E)-3-(3,7-Dimethyl-1,4-dioxy-quinoxalin-2-yl)-1-(3,4,5-trimethoxy-phenyl)-propenone (4e). The compound 4e was obtained as yellow solid (33\%). IR $(\mathrm{KBr}) 1654(\mathrm{C}=\mathrm{O}), 1322(\mathrm{~N}-\mathrm{O}), 1126(\mathrm{C}-\mathrm{O}-\mathrm{C}) ;{ }^{1} \mathrm{H}-\mathrm{NMR}\left(\mathrm{CDCl}_{3}\right) \delta: 2.66(\mathrm{~s}$, $\left.3 \mathrm{H}, 7-\mathrm{CH}_{3}\right), 2.94\left(\mathrm{~s}, 3 \mathrm{H}, 3-\mathrm{CH}_{3}\right), 3.99\left(\mathrm{~s}, 3 \mathrm{H}, p-\mathrm{OCH}_{3}\right), 4.00\left(\mathrm{~s}, 6 \mathrm{H}, m-\mathrm{OCH}_{3}\right), 7.41\left(\mathrm{~s}, 2 \mathrm{H}, \mathrm{H}_{2}{ }^{\prime}+\mathrm{H}_{6}{ }^{\prime}\right), 7.71-7.73(\mathrm{dd}$, $\left.1 \mathrm{H}, \mathrm{H}_{6}, J_{65}=8.74 \mathrm{~Hz}, J_{68}=1.62 \mathrm{~Hz}\right), 7.84-7.88\left(\mathrm{~d}, 1 \mathrm{H}, \mathrm{H}_{\mathrm{b}}, J_{\mathrm{ba}}=15.27 \mathrm{~Hz}\right), 8.45\left(\mathrm{~s}, 1 \mathrm{H}, \mathrm{H}_{8}\right), 8.56-8.59\left(\mathrm{~d}, 1 \mathrm{H}, \mathrm{H}_{5}\right.$, $\left.J_{56}=8.75 \mathrm{~Hz}\right), 9.14-9.18\left(\mathrm{~d}, 1 \mathrm{H}, \mathrm{H}_{\mathrm{b}}, J_{\mathrm{ba}}=15.29 \mathrm{~Hz}\right)$; Analysis calculated for $\mathrm{C}_{22} \mathrm{H}_{22} \mathrm{~N}_{2} \mathrm{O}_{6}(410.42): \mathrm{C}, 64.39 ; \mathrm{H}, 5.37$; N, 6.83. Found: C, 64.19; H, 5.40; N, 6.64.

(2E)-1-(3-Hydroxy-4,5-dimethoxy-phenyl)-3-(3,6,7-trimethyl-1,4-dioxy-quinoxalin-2-yl)-propenone (4f). The compound $\mathbf{4 f}$ was obtained as yellow solid $(36 \%)$. IR ( KBr) $1654(\mathrm{C}=\mathrm{O}), 1321(\mathrm{~N}-\mathrm{O}), 1128(\mathrm{C}-\mathrm{O}-\mathrm{C})$; ${ }^{1} \mathrm{H}-\mathrm{NMR}$ $\left(\mathrm{CDCl}_{3}\right) \delta: 2.55\left(\mathrm{~s}, 6 \mathrm{H}, 6,7-\mathrm{CH}_{3}\right), 2.93\left(\mathrm{~s}, 3 \mathrm{H}, 3-\mathrm{CH}_{3}\right), 3.98\left(\mathrm{~s}, 3 \mathrm{H}, \mathrm{p}-\mathrm{OCH}_{3}\right), 3.99\left(\mathrm{~s}, 6 \mathrm{H}, \mathrm{m}-\mathrm{OCH}_{3}\right), 7.40(\mathrm{~s}, 2 \mathrm{H}$, $\left.\mathrm{H}_{2}{ }^{\prime}+\mathrm{H}_{6}{ }^{\prime}\right), 7.83-7.87\left(\mathrm{~d}, 1 \mathrm{H}, \mathrm{H}_{\mathrm{b}}, J_{\mathrm{ba}}=15.27 \mathrm{~Hz}\right), 8.39\left(\mathrm{~s}, 1 \mathrm{H}, \mathrm{H}_{5}\right), 8.42\left(\mathrm{~s}, 1 \mathrm{H}, \mathrm{H}_{8}\right) 9.13-9.17\left(\mathrm{~d}, 1 \mathrm{H}, \mathrm{H}_{\mathrm{a}}, J_{\mathrm{ab}}=15.28 \mathrm{~Hz}\right)$; Analysis calculated for $\mathrm{C}_{23} \mathrm{H}_{24} \mathrm{~N}_{2} \mathrm{O}_{6}$ (424.45): C, 65.08; H, 5.70; N, 6.60. Found: C, 65.16; H, 5.57; N, 6.46.

(2E)- 3-(1,4-Dioxy-quinoxalin-2-yl)-1-(4-methoxy-phenyl)-propenone (5a). The derivative 5a was obtained as yellow solid (46\%). IR (KBr) $1663(\mathrm{C}=\mathrm{O}), 1374(\mathrm{~N}-\mathrm{O}), 1174(\mathrm{C}-\mathrm{O}-\mathrm{C}) ;{ }^{1} \mathrm{H}-\mathrm{NMR}\left(\mathrm{DMSO}_{6}\right)$ 8: $3.90\left(\mathrm{~s}, 3 \mathrm{H}, \mathrm{OCH}_{3}\right)$, 7.12-7.15 (d, $\left.2 \mathrm{H}, \mathrm{H}_{2}{ }^{\prime}+\mathrm{H}_{6}{ }^{\prime}, J_{2}{ }^{\prime}{ }^{\prime} / J_{6}{ }^{\prime}{ }^{\prime}=8.78 \mathrm{~Hz}\right), 7.98-8.01\left(\mathrm{~m}, 2 \mathrm{H}, \mathrm{H}_{6}+\mathrm{H}_{7}\right), 8.05-8.09\left(\mathrm{~d}, 1 \mathrm{H}, \mathrm{H}_{\mathrm{b}}, J_{\mathrm{ba}}=15.93 \mathrm{~Hz}\right), 8.22-$ $8.24\left(\mathrm{~d}, 2 \mathrm{H}, \mathrm{H}_{3}{ }^{\prime}+\mathrm{H}_{5}{ }^{\prime}, J_{3}{ }^{\prime}{ }^{\prime} / J_{5}{ }^{\prime}{ }^{\prime}=8.70 \mathrm{~Hz}\right), 8.47-8.54\left(\mathrm{~m}, 2 \mathrm{H}, \mathrm{H}_{5}+\mathrm{H}_{8}\right), 8.58-8.62\left(\mathrm{~d}, 1 \mathrm{H}, \mathrm{H}_{\mathrm{a}}, J_{\mathrm{ab}}=15.95 \mathrm{~Hz}\right), 9.53$ (s, $1 \mathrm{H}, \mathrm{H}_{3}$ ); Analysis calculated for $\mathrm{C}_{18} \mathrm{H}_{14} \mathrm{~N}_{2} \mathrm{O}_{4}$ (322.32): C, 67.08; H, 4.35; N, 8.70. Found: C, 67.14; H, 4.43; N, 8.71.

(2E)-3-(7-Fluoro-1,4-dioxy-quinoxalin-2-yl)-1-(4-methoxy-phenyl)-propenone (5b). The compound $5 \mathbf{b}$ was obtained as yellow solid (32\%). IR (KBr) $1661(\mathrm{C}=\mathrm{O}), 1372(\mathrm{~N}-\mathrm{O}), 1177(\mathrm{C}-\mathrm{O}-\mathrm{C}) ;{ }^{1} \mathrm{H}-\mathrm{NMR}\left(\mathrm{DMSO}-\mathrm{d}_{6}\right)$ 8: $3.89(\mathrm{~s}$, $\left.3 \mathrm{H}, \mathrm{OCH}_{3}\right), 7.13-7.15\left(\mathrm{~d}, 2 \mathrm{H}, \mathrm{H}_{2}{ }^{\prime}+\mathrm{H}_{6}{ }^{\prime}, J_{2}{ }^{\prime}{ }^{\prime} / J_{6}{ }^{\prime}{ }^{\prime}=8.83 \mathrm{~Hz}\right), 7.90-7.95\left(\mathrm{~m}, 1 \mathrm{H}, \mathrm{H}_{6}\right), 8.02-8.06\left(\mathrm{~d}, 1 \mathrm{H}, \mathrm{H}_{\mathrm{b}}, J_{\mathrm{ba}}=15.96\right.$ $\mathrm{Hz}), 8.22-8.24\left(\mathrm{~m}, 3 \mathrm{H}, \mathrm{H}_{8}+\mathrm{H}_{3}{ }^{\prime}+\mathrm{H}_{5}{ }^{\prime}\right), 8.58-8.62\left(\mathrm{~m}, 2 \mathrm{H}, \mathrm{H}_{\mathrm{a}}+\mathrm{H}_{5}\right), 9.59\left(\mathrm{~s}, 1 \mathrm{H}, \mathrm{H}_{3}\right)$; Analysis calculated for $\mathrm{C}_{18} \mathrm{H}_{13} \mathrm{FN}_{2} \mathrm{O}_{4}$ (340.31): C, 63.53; H, 3.82; N, 8.24. Found: C, 63.29; H, 3.72; N, 8.02.

(2E)-3-(7-Chloro-1,4-dioxy-quinoxalin-2-yl)-1-(4-methoxy-phenyl)-propenone (5d). The derivative 5d was obtained as yellow solid (71\%). IR (KBr) $1661(\mathrm{C}=\mathrm{O}), 1374(\mathrm{~N}-\mathrm{O}), 1177(\mathrm{C}-\mathrm{O}-\mathrm{C})$; ${ }^{1} \mathrm{H}-\mathrm{NMR}\left(\mathrm{DMSO}-\mathrm{d}_{6}\right)$ 8: $3.89(\mathrm{~s}$, $\left.3 \mathrm{H}, \mathrm{OCH}_{3}\right), 7.13-7.15\left(\mathrm{~d}, 2 \mathrm{H}, \mathrm{H}_{2}{ }^{\prime}+\mathrm{H}_{6}{ }^{\prime}, J_{2}{ }^{3}{ }^{\prime} / J_{6}{ }^{\circ}{ }^{\prime}=8.78 \mathrm{~Hz}\right), 8.02-8.06\left(\mathrm{~m}, 2 \mathrm{H}, \mathrm{H}_{6}+\mathrm{H}_{\mathrm{b}}\right), 8.21-8.23\left(\mathrm{~d}, 2 \mathrm{H}_{,} \mathrm{H}_{3}{ }^{\circ}+\mathrm{H}_{5}{ }^{\prime}\right.$, $\left.J_{3^{\prime},}, / J_{5^{\prime}}{ }^{\prime}=8.27 \mathrm{~Hz}\right), 8.47-8.63\left(\mathrm{~m}, 3 \mathrm{H}, \mathrm{H}_{\mathrm{a}}+\mathrm{H}_{5}+\mathrm{H}_{8}\right), 9.58\left(\mathrm{~s}, 1 \mathrm{H}, \mathrm{H}_{3}\right)$; Analysis calculated for $\mathrm{C}_{18} \mathrm{H}_{13} \mathrm{ClN}_{2} \mathrm{O}_{4}(356.77)$ : C, 60.59; H, 3.65; N, 7.85. Found: C, 60.23; H, 3.52; N, 7.64.

(2E)-1-(4-Methoxy-phenyl)-3-(7-methyl-1,4-dioxy-quinoxalin-2-yl)-propenone (5e). The compound 5e was obtained as yellow solid (39\%). IR ( KBr) $1657(\mathrm{C}=\mathrm{O}), 1373(\mathrm{~N}-\mathrm{O}), 1171(\mathrm{C}-\mathrm{O}-\mathrm{C}) ;{ }^{1} \mathrm{H}-\mathrm{NMR}\left(\mathrm{DMSO}-\mathrm{d}_{6}\right) \delta: 2.60(\mathrm{~s}$, $\left.3 \mathrm{H}, \mathrm{CH}_{3}\right), 3.90\left(\mathrm{~s}, 3 \mathrm{H}, \mathrm{OCH}_{3}\right), 7.13-7.15\left(\mathrm{~d}, 2 \mathrm{H}, \mathrm{H}_{2}{ }^{\prime}+\mathrm{H}_{6}{ }^{\prime}, J_{2}{ }^{\prime}{ }^{\prime} / J_{6}{ }^{\prime}{ }^{\prime}=8.91 \mathrm{~Hz}\right), 7.82-7.84\left(\mathrm{dd}, 1 \mathrm{H}, \mathrm{H}_{6}, J_{65}=8.81 \mathrm{~Hz}\right.$, $\left.J_{68}=1.74 \mathrm{~Hz}\right), 8.04-8.08\left(\mathrm{~d}, 1 \mathrm{H}, \mathrm{H}_{\mathrm{b}}, \mathrm{J}_{\mathrm{ba}}=15.96 \mathrm{~Hz}\right), 8.22-8.24\left(\mathrm{~d}, 2 \mathrm{H}, \mathrm{H}_{3}{ }^{\prime}+\mathrm{H}_{5}{ }^{\prime}, J_{3^{\prime}{ }^{\prime}}, J_{5^{\prime} 6^{\prime}}=8.94 \mathrm{~Hz}\right), 8.30\left(\mathrm{~s}, 1 \mathrm{H}, \mathrm{H}_{8}\right)$, 8.41-8.43 (d, $\left.1 \mathrm{H}, \mathrm{H}_{5}, J_{56}=8.76 \mathrm{~Hz}\right), 8.56-8.60\left(\mathrm{~d}, 1 \mathrm{H}, \mathrm{H}_{\mathrm{a}}, J_{\mathrm{ab}}=15.97 \mathrm{~Hz}\right), 9.50\left(\mathrm{~s}, 1 \mathrm{H}, \mathrm{H}_{3}\right)$; Analysis calculated for $\mathrm{C}_{19} \mathrm{H}_{16} \mathrm{~N}_{2} \mathrm{O}_{4}$ (336.35): C, 67.86; H, 4.76; N, 8.33. Found: $\mathrm{C}, 67.68 ; \mathrm{H}, 4.51 ; \mathrm{N}, 8.55$.

(2E)-3-(6,7-Difluoro-1,4-dioxy-quinoxalin-2-yl)-1-(4-methoxy-phenyl)-propenone (5g). The compound $5 g$ was obtained as yellow solid (14\%). IR (KBr) $1662(\mathrm{C}=\mathrm{O}), 1374(\mathrm{~N}-\mathrm{O}), 1176(\mathrm{C}-\mathrm{O}-\mathrm{C})$; ${ }^{1} \mathrm{H}-\mathrm{NMR}\left(\mathrm{DMSO}-\mathrm{d}_{6}\right)$ \&: $3.90(\mathrm{~s}$, 
$3 \mathrm{H}, \mathrm{OCH}_{3}$ ), 7.14-7.16 (d, 2H, $\left.\mathrm{H}_{2}{ }^{\prime}+\mathrm{H}_{6}{ }^{\prime}, J_{2}{ }^{3}, J_{6}{ }^{\circ},=8.68 \mathrm{~Hz}\right), 8.01-8.05\left(\mathrm{~d}, 1 \mathrm{H}, \mathrm{H}_{\mathrm{b}}, J_{\mathrm{ba}}=16.03 \mathrm{~Hz}\right), 8.22-8.24(\mathrm{~d}, 2 \mathrm{H}$,

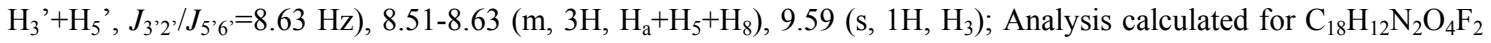
(358.30): C, 60.34; H, 3.38; N, 7.82. Found: C, 60.07; H, 3.35; N, 7.65.

General method for the synthesis of (2E)-1-(3-Methyl-quinoxalin-2-yl)-3-(3,4,5-trimethoxy-phenyl)-propenone derivatives (Series 6). The synthesis of compounds 6 was carried out as shown in Scheme 2. The starting reagents used (VII and VIII) were obtained by means of previously described methods. ${ }^{23,24}$ To a solution of 1-(3-Methylquinoxalin-2-yl)-ethanone derivative VIII $(1 \mathrm{mmol})$ and 3,4,5-Trimethoxy-benzaldehyde $(1 \mathrm{mmol})$ in methanol, a solution of $3 \% \mathrm{NaOH}$ in methanol $(1 \mathrm{~mL})$ was added. After 24 hours, the reaction mixture was filtered and the solid washed with water.

(2E)-1-(3-Methyl-quinoxalin-2-yl)-3-(3,4,5-trimethoxy-phenyl)-propenone (6a). The derivative 6a was obtained as yellow solid (56\%). IR (KBr) $1666(\mathrm{C}=\mathrm{O}), 1133(\mathrm{C}-\mathrm{O}-\mathrm{C}) ;{ }^{1} \mathrm{H}-\mathrm{NMR}\left(\mathrm{CDCl}_{3}\right)$ 8: $3.01\left(\mathrm{~s}, 3 \mathrm{H}, \mathrm{CH}_{3}\right), 3.94(\mathrm{~s}, 3 \mathrm{H}, p$ $\left.\mathrm{OCH}_{3}\right), 3.95\left(\mathrm{~s}, 6 \mathrm{H}, \mathrm{m}-\mathrm{OCH}_{3}\right), 6.94\left(\mathrm{~s}, 2 \mathrm{H}, \mathrm{H}_{2}{ }^{\prime}+\mathrm{H}_{6}{ }^{\prime}\right), 7.75-7.79\left(\mathrm{~d}, 1 \mathrm{H}, \mathrm{H}_{\mathrm{a}}, J_{\mathrm{ab}}=16.02 \mathrm{~Hz}\right), 7.81-7.90\left(\mathrm{~m}, 2 \mathrm{H}, \mathrm{H}_{6}+\mathrm{H}_{7}\right)$, 7.84-7.88 (d, $\left.1 \mathrm{H}, \mathrm{H}_{\mathrm{b}}, J_{\mathrm{ba}}=15.94 \mathrm{~Hz}\right), 8.09-8.11\left(\mathrm{~d}, 1 \mathrm{H}, \mathrm{H}_{5}, J_{56}=8.31 \mathrm{~Hz}\right), 8.20-8.22\left(\mathrm{~d}, 1 \mathrm{H}, \mathrm{H}_{8}, J_{87}=8.30 \mathrm{~Hz}\right)$; Analysis calculated for $\mathrm{C}_{21} \mathrm{H}_{20} \mathrm{~N}_{2} \mathrm{O}_{4}$ (364.40): C, 69.23; H, 5.49; N, 7.69. Found: C, 69.46; H, 5.53; N, 7.45.

(2E)-1-(7-Fluoro-3-methyl-quinoxalin-2-yl)-3-(3,4,5-trimethoxy-phenyl)-propenone (6b). The compound $6 \mathbf{b}$ was obtained as yellow solid (59\%). IR (KBr) $1670(\mathrm{C}=\mathrm{O}), 1128(\mathrm{C}-\mathrm{O}-\mathrm{C}) ;{ }^{1} \mathrm{H}-\mathrm{NMR}\left(\mathrm{CDCl}_{3}\right) \delta: 2.98\left(\mathrm{~s}, 3 \mathrm{H}, \mathrm{CH}_{3}\right), 3.94$ (s, $\left.3 \mathrm{H}, p-\mathrm{OCH}_{3}\right), 3.95\left(\mathrm{~s}, 6 \mathrm{H}, m-\mathrm{OCH}_{3}\right), 6.93\left(\mathrm{~s}, 2 \mathrm{H}, \mathrm{H}_{2}{ }^{\prime}+\mathrm{H}_{6}\right.$ '), 7.63-7.68 (ddd, $1 \mathrm{H}, \mathrm{H}_{6}, J_{65}=9.26 \mathrm{~Hz}, J_{6 \mathrm{~F}}=8.12 \mathrm{~Hz}$, $\left.J_{68}=2.83 \mathrm{~Hz}\right), 7.74-7.78\left(\mathrm{~d}, 1 \mathrm{H}, \mathrm{H}_{\mathrm{a}}, J_{\mathrm{ab}}=15.98 \mathrm{~Hz}\right), 7.79-7.83\left(\mathrm{~d}, 1 \mathrm{H}, \mathrm{H}_{\mathrm{b}}, J_{\mathrm{ba}}=15.97 \mathrm{~Hz}\right), 7.82-7.85\left(\mathrm{dd}, 1 \mathrm{H}, \mathrm{H}_{8}\right.$, $\left.J_{8 \mathrm{~F}}=8.84 \mathrm{~Hz}, \mathrm{~J}_{86}=2.78 \mathrm{~Hz}\right), 8.09-8.12\left(\mathrm{dd}, 1 \mathrm{H}, \mathrm{H}_{5}, J_{56}=9.25 \mathrm{~Hz}, J_{5 \mathrm{~F}}=5.60 \mathrm{~Hz}\right)$; Analysis calculated for $\mathrm{C}_{21} \mathrm{H}_{19} \mathrm{~N}_{2} \mathrm{O}_{4} \mathrm{~F}$ (382.39): C, 65.97; H, 4.97; N, 7.33. Found: C, 66.13; H, 5.04; N, 7.11.

(2E)-1-(7-Methoxy-3-methyl-quinoxalin-2-yl)-3-(3,4,5-trimethoxy-phenyl)-propenone (6c). The derivative 6c was obtained as yellow solid (31\%). IR ( $\mathrm{KBr}) 1670(\mathrm{C}=\mathrm{O}), 1126(\mathrm{C}-\mathrm{O}-\mathrm{C}) ;{ }^{1} \mathrm{H}-\mathrm{NMR}\left(\mathrm{CDCl}_{3}\right) \delta: 2.97\left(\mathrm{~s}, 3 \mathrm{H}, \mathrm{CH}_{3}\right)$, $3.94\left(\mathrm{~s}, 3 \mathrm{H}, p-\mathrm{OCH}_{3}\right), 3.95\left(\mathrm{~s}, 6 \mathrm{H}, m-\mathrm{OCH}_{3}\right), 4.02\left(\mathrm{~s}, 3 \mathrm{H}\right.$, quinox- $\left.-\mathrm{OCH}_{3}\right), 6.93\left(\mathrm{~s}, 2 \mathrm{H}, \mathrm{H}_{2}{ }^{\prime}+\mathrm{H}_{6}{ }^{\prime}\right), 7.47-7.48\left(\mathrm{~d}, 1 \mathrm{H}, \mathrm{H}_{8}\right.$, $\left.J_{86}=2.61 \mathrm{~Hz}\right), 7.51-7.54\left(\mathrm{dd}, 1 \mathrm{H}, \mathrm{H}_{6}, J_{65}=9.17 \mathrm{~Hz}, J_{68}=2.75 \mathrm{~Hz}\right), 7.73-7.77\left(\mathrm{~d}, 1 \mathrm{H}, \mathrm{H}_{\mathrm{a}}, J_{\mathrm{ab}}=16.00 \mathrm{~Hz}\right), 7.80-7.84(\mathrm{~d}$, $\left.1 \mathrm{H}, \mathrm{H}_{\mathrm{b}}, J_{\mathrm{ba}}=15.98 \mathrm{~Hz}\right), 7.99-8.02\left(\mathrm{~d}, 1 \mathrm{H}, \mathrm{H}_{5}, J_{56}=9.17 \mathrm{~Hz}\right)$; Analysis calculated for $\mathrm{C}_{22} \mathrm{H}_{22} \mathrm{~N}_{2} \mathrm{O}_{5}(394.43): \mathrm{C}, 67.00 ; \mathrm{H}$, 5.58; N, 7.11. Found: C, 67.08; H, 5.61; N, 7.05.

(2E)-1-(7-Chloro-3-methyl-quinoxalin-2-yl)-3-(3,4,5-trimethoxy-phenyl)-propenone (6d). The derivative 6d was obtained as yellow solid (31\%). IR (KBr) $1670(\mathrm{C}=\mathrm{O}), 1128(\mathrm{C}-\mathrm{O}-\mathrm{C}) ;{ }^{1} \mathrm{H}-\mathrm{NMR}\left(\mathrm{CDCl}_{3}\right) \delta: 2.99\left(\mathrm{~s}, 3 \mathrm{H}, \mathrm{CH}_{3}\right), 3.94$ (s, $\left.3 \mathrm{H}, p-\mathrm{OCH}_{3}\right), 3.96\left(\mathrm{~s}, 6 \mathrm{H}, m-\mathrm{OCH}_{3}\right), 6.93\left(\mathrm{~s}, 2 \mathrm{H}, \mathrm{H}_{2}{ }^{\prime}+\mathrm{H}_{6}{ }^{\prime}\right), 7.74-7.78\left(\mathrm{~d}, 1 \mathrm{H}, \mathrm{H}_{\mathrm{a}}, J_{\mathrm{ab}}=16.00 \mathrm{~Hz}\right), 7.79-7.83(\mathrm{~d}, 1 \mathrm{H}$, $\left.\mathrm{H}_{\mathrm{b}}, J_{\mathrm{ba}}=15.98 \mathrm{~Hz}\right), 7.79-7.82\left(\mathrm{dd}, 1 \mathrm{H}, \mathrm{H}_{6}, J_{65}=8.93 \mathrm{~Hz}, J_{68}=2.26 \mathrm{~Hz}\right), 8.03-8.05\left(\mathrm{~d}, 1 \mathrm{H}, \mathrm{H}_{5}, J_{56}=8.96 \mathrm{~Hz}\right), 8.21-8.22$ $\left(\mathrm{d}, 1 \mathrm{H}, \mathrm{H}_{8}, J_{86}=2.21 \mathrm{~Hz}\right.$ ); Analysis calculated for $\mathrm{C}_{21} \mathrm{H}_{19} \mathrm{~N}_{2} \mathrm{O}_{4} \mathrm{Cl}$ (398.85): C, 63.24; H, 4.77; N, 7.03. Found: C, $62.92 ; \mathrm{H}, 4.89 ; \mathrm{N}, 6.73$.

(2E)-1-(3,7-Dimethyl-quinoxalin-2-yl)-3-(3,4,5-trimethoxy-phenyl)-propenone (6e). The compound 6e was obtained as yellow solid (49\%). IR (KBr) $1670(\mathrm{C}=\mathrm{O}), 1126(\mathrm{C}-\mathrm{O}-\mathrm{C}) ;{ }^{1} \mathrm{H}-\mathrm{NMR}\left(\mathrm{CDCl}_{3}\right) \delta: 2.64\left(\mathrm{~s}, 3 \mathrm{H}, 7-\mathrm{CH}_{3}\right), 3.00$ $\left(\mathrm{s}, 3 \mathrm{H}, 3-\mathrm{CH}_{3}\right), 3.94\left(\mathrm{~s}, 3 \mathrm{H}, \mathrm{p}-\mathrm{OCH}_{3}\right), 3.95\left(\mathrm{~s}, 6 \mathrm{H}, \mathrm{m}-\mathrm{OCH}_{3}\right), 6.93\left(\mathrm{~s}, 2 \mathrm{H}, \mathrm{H}_{2}{ }^{\prime}+\mathrm{H}_{6}{ }^{\prime}\right), 7.69-7.72\left(\mathrm{dd}, 1 \mathrm{H}, \mathrm{H}_{6}, J_{65}=8.55\right.$ $\left.\mathrm{Hz}, J_{68}=1.76 \mathrm{~Hz}\right), 7.74-7.78\left(\mathrm{~d}, 1 \mathrm{H}, \mathrm{H}_{\mathrm{a}}, J_{\mathrm{ab}}=15.99 \mathrm{~Hz}\right), 7.85-7.89\left(\mathrm{~d}, 1 \mathrm{H}, \mathrm{H}_{\mathrm{b}}, J_{\mathrm{ba}}=15.94 \mathrm{~Hz}\right), 7.98\left(\mathrm{~s}, 1 \mathrm{H}, \mathrm{H}_{8}\right), 7.99-$ $8.01\left(\mathrm{~d}, 1 \mathrm{H}, \mathrm{H}_{5}, J_{56}=8.75 \mathrm{~Hz}\right.$ ); Analysis calculated for $\mathrm{C}_{22} \mathrm{H}_{22} \mathrm{~N}_{2} \mathrm{O}_{4}$ (378.43): C, 69.84; H, 5.82; N, 7.41. Found: C, 69.92; H, 5.79; N, 7.48.

(2E)-3-(3,4,5-Trimethoxy-phenyl)-1-(3,6,7-trimethyl-quinoxalin-2-yl)-propenone (6f). The compound 6 ff was obtained as yellow solid (38\%). IR ( $\mathrm{KBr}) 1671(\mathrm{C}=\mathrm{O}), 1127(\mathrm{C}-\mathrm{O}-\mathrm{C}) ;{ }^{1} \mathrm{H}-\mathrm{NMR}\left(\mathrm{CDCl}_{3}\right) \delta$ : $2.55\left(\mathrm{~s}, 3 \mathrm{H}, 6 / 7-\mathrm{CH}_{3}\right)$, $2.56\left(\mathrm{~s}, 3 \mathrm{H}, 6 / 7-\mathrm{CH}_{3}\right), 3.01\left(\mathrm{~s}, 3 \mathrm{H}, 3-\mathrm{CH}_{3}\right), 3.94\left(\mathrm{~s}, 3 \mathrm{H}, p-\mathrm{OCH}_{3}\right), 3.96\left(\mathrm{~s}, 6 \mathrm{H}, m-\mathrm{OCH}_{3}\right), 6.94\left(\mathrm{~s}, 2 \mathrm{H}, \mathrm{H}_{2}{ }^{\prime}+\mathrm{H}_{6}{ }^{\prime}\right), 7.75-$ $7.79\left(\mathrm{~d}, 1 \mathrm{H}, \mathrm{H}_{\mathrm{a}}, J_{\mathrm{ab}}=15.96 \mathrm{~Hz}\right), 7.89\left(\mathrm{~s}, 1 \mathrm{H}, \mathrm{H}_{8}\right), 7.89-7.93\left(\mathrm{~d}, 1 \mathrm{H}, \mathrm{H}_{\mathrm{b}}, J_{\mathrm{ba}}=15.95 \mathrm{~Hz}\right), 7.96\left(\mathrm{~s}, 1 \mathrm{H}, \mathrm{H}_{5}\right)$; Analysis calculated for $\mathrm{C}_{23} \mathrm{H}_{24} \mathrm{~N}_{2} \mathrm{O}_{4}$ (392.46): C, 70.41; H, 6.12; N, 7.14. Found: C, 70.34; H, 6.12; N, 7.00.

General method for the synthesis of 4-(3-Methyl-quinoxalin-2-yl)-6-(3,4,5-trimethoxy-phenyl)-pyrimidin-2ylamine derivatives (Series 7). To a solution of the corresponding compound of series 6 ( $0.5 \mathrm{mmol})$ and guanidine hydrochloride $(1 \mathrm{mmol})$ in isopropanol, $0.56 \mathrm{~mL}$ of a solution $10 \% \mathrm{KOH}$ in isopropanol were added. The reaction mixture was refluxing over 24 hours. The solvent was removed under reduced pressure and the crude residue was purified by flash chromatography using hexane:ethyl acetate (50:50) to yield compounds 7.

4-(3-Methyl-quinoxalin-2-yl)-6-(3,4,5-trimethoxy-phenyl)-pyrimidin-2-ylamine (7a). The derivative 7a was obtained as beige solid (15\%). IR (KBr) 3432-3346 (N-H), $1620(\mathrm{C}=\mathrm{N}), 1126(\mathrm{C}-\mathrm{O}-\mathrm{C}) ;{ }^{1} \mathrm{H}-\mathrm{NMR}\left(\mathrm{DMSO}-\mathrm{d}_{6}\right) \delta: 2.84$ $\left(\mathrm{s}, 3 \mathrm{H}, \mathrm{CH}_{3}\right) ; 3.75\left(\mathrm{~s}, 3 \mathrm{H}, p-\mathrm{OCH}_{3}\right) ; 3.88\left(\mathrm{~s}, 6 \mathrm{H}, \mathrm{m}-\mathrm{OCH}_{3}\right) ; 6.96\left(\mathrm{~s}, 2 \mathrm{H}, \mathrm{NH}_{2}\right) ; 7.48\left(\mathrm{~s}, 2 \mathrm{H}, \mathrm{H}_{2}{ }^{\prime}+\mathrm{H}_{6}{ }^{\prime}\right) ; 7.65(\mathrm{~s}, 1 \mathrm{H}$, ar$\mathrm{CH})$; 7.84-7.92 (m, $\left.2 \mathrm{H}, \mathrm{H}_{6}+\mathrm{H}_{7}\right)$; 8.08-8.11 (dd, $\left.1 \mathrm{H}, \mathrm{H}_{8}, J_{87}=8.24 \mathrm{~Hz} ; J_{86}=1.22 \mathrm{~Hz}\right) ; 8.15-8.17\left(\mathrm{~d}, 1 \mathrm{H}, \mathrm{H}_{5}, J_{56}=8.27\right.$ $\mathrm{Hz}$ ); Analysis calculated for $\mathrm{C}_{22} \mathrm{H}_{21} \mathrm{~N}_{5} \mathrm{O}_{3}$ (403.44): C, 65.51; H, 5.21; N, 17.37. Found: C, 65.59; H, 5.45; N, 17.48.

4-(7-Fluoro-3-methyl-quinoxalin-2-yl)-6-(3,4,5-trimethoxy-phenyl)-pyrimidin-2-ylamine (7b). The compound 7b was obtained as beige solid (15\%). IR (KBr) 3430-3308 (N-H), $1628(\mathrm{C}=\mathrm{N}), 1133(\mathrm{C}-\mathrm{O}-\mathrm{C}) ;{ }^{1} \mathrm{H}-\mathrm{NMR}\left(\mathrm{DMSO}^{\left.-\mathrm{d}_{6}\right)}\right.$ 
$\delta: 2.83\left(\mathrm{~s}, 3 \mathrm{H}, \mathrm{CH}_{3}\right) ; 3.74\left(\mathrm{~s}, 3 \mathrm{H}, p-\mathrm{OCH}_{3}\right) ; 3.88\left(\mathrm{~s}, 6 \mathrm{H}, m-\mathrm{OCH}_{3}\right) ; 6.98\left(\mathrm{~s}, 2 \mathrm{H}, \mathrm{NH}_{2}\right) ; 7.47\left(\mathrm{~s}, 2 \mathrm{H}, \mathrm{H}_{2}{ }^{\prime}+\mathrm{H}_{6}{ }^{\prime}\right) ; 7.65(\mathrm{~s}$, $1 \mathrm{H}$, ar- $\mathrm{CH}) ; 7.81-7.86\left(\mathrm{ddd}, 1 \mathrm{H}, \mathrm{H}_{6}, J_{65}=9.13 \mathrm{~Hz} ; J_{6 \mathrm{~F}}=8.86 \mathrm{~Hz} ; J_{68}=2.81 \mathrm{~Hz}\right) ; 7.94-7.97\left(\mathrm{dd}, 1 \mathrm{H}, \mathrm{H}_{8}, J_{8 \mathrm{~F}}=9.37 \mathrm{~Hz}\right.$; $\left.J_{86}=2.78 \mathrm{~Hz}\right) ; 8.16-8.20\left(\mathrm{dd}, 1 \mathrm{H}, \mathrm{H}_{5}, J_{56}=9.23 \mathrm{~Hz} ; J_{5 \mathrm{~F}}=5.84 \mathrm{~Hz}\right.$ ); Analysis calculated for $\mathrm{C}_{22} \mathrm{H}_{20} \mathrm{FN}_{5} \mathrm{O}_{3}(421.43)$ : C, $62.71 ; \mathrm{H}, 4.75 ; \mathrm{N}, 16.63$. Found: C, 62.30; H, 5.15; N, 16.40 .

4-(7-Methoxy-3-methyl-quinoxalin-2-yl)-6-(3,4,5-trimethoxy-phenyl)-pyrimidin-2-ylamine (7c). The derivative 7c was obtained as beige solid (9\%). IR (KBr) 3458-3212 (N-H), $1620(\mathrm{C}=\mathrm{N}), 1127(\mathrm{C}-\mathrm{O}-\mathrm{C}) ;{ }^{1} \mathrm{H}-\mathrm{NMR}\left(\mathrm{DMSO}_{6} \mathrm{~d}_{6}\right)$ $\delta: 2.79\left(\mathrm{~s}, 3 \mathrm{H}, \mathrm{CH}_{3}\right) ; 3.74\left(\mathrm{~s}, 3 \mathrm{H}, p-\mathrm{OCH}_{3}\right) ; 3.88\left(\mathrm{~s}, 6 \mathrm{H}, m-\mathrm{OCH}_{3}\right) ; 3.96\left(\mathrm{~s}, 3 \mathrm{H}, 7-\mathrm{OCH}_{3}\right) ; 6.94\left(\mathrm{~s}, 2 \mathrm{H}, \mathrm{NH}_{2}\right) ; 7.48(\mathrm{~s}$, $\left.2 \mathrm{H}, \mathrm{H}_{2}{ }^{\prime}+\mathrm{H}_{6}{ }^{\prime}\right)$; 7.51-7.54 (m, 2H, $\left.\mathrm{H}_{6}+\mathrm{H}_{8}\right) ; 7.63(\mathrm{~s}, 1 \mathrm{H}$, ar- $\mathrm{CH})$; 7.97-8.00 (d, $\left.1 \mathrm{H}, \mathrm{H}_{5}, J_{56}=9.92 \mathrm{~Hz}\right)$; Analysis calculated for $\mathrm{C}_{23} \mathrm{H}_{23} \mathrm{~N}_{5} \mathrm{O}_{4}$ (433.47): C, 63.74; H, 5.31; N, 16.17. Found: $\mathrm{C}, 63.35 ; \mathrm{H}, 5.39 ; \mathrm{N}, 15.99$.

4-(7-Chloro-3-methyl-quinoxalin-2-yl)-6-(3,4,5-trimethoxy-phenyl)-pyrimidin-2-ylamine (7d). The derivative 7d was obtained as beige solid $(10 \%)$. IR $(\mathrm{KBr}) 3447-3308(\mathrm{~N}-\mathrm{H}), 1630(\mathrm{C}=\mathrm{N}), 1132(\mathrm{C}-\mathrm{O}-\mathrm{C}) ;{ }^{1} \mathrm{H}-\mathrm{NMR}\left(\mathrm{DMSO}_{6}\right) \delta$ : $2.84\left(\mathrm{~s}, 3 \mathrm{H}, \mathrm{CH}_{3}\right) ; 3.74\left(\mathrm{~s}, 3 \mathrm{H}, p-\mathrm{OCH}_{3}\right) ; 3.88\left(\mathrm{~s}, 6 \mathrm{H}, \mathrm{m}-\mathrm{OCH}_{3}\right) ; 6.99\left(\mathrm{~s}, 2 \mathrm{H}, \mathrm{NH}_{2}\right) ; 7.47\left(\mathrm{~s}, 2 \mathrm{H}, \mathrm{H}_{2}{ }^{\prime}+\mathrm{H}_{6}{ }^{\prime}\right) ; 7.65(\mathrm{~s}$, $1 \mathrm{H}$, ar-CH); 7.91-7.94 (dd, $\left.1 \mathrm{H}, \mathrm{H}_{6}, J_{65}=8.93 \mathrm{~Hz} ; J_{68}=2.38 \mathrm{~Hz}\right) ; 8.12-8.14\left(\mathrm{~d}, 1 \mathrm{H}, \mathrm{H}_{5}, J_{56}=8.95 \mathrm{~Hz}\right) ; 8.26-8.27(\mathrm{~d}, 1 \mathrm{H}$, $\mathrm{H}_{8}, J_{86}=2.26 \mathrm{~Hz}$ ); Analysis calculated for $\mathrm{C}_{22} \mathrm{H}_{20} \mathrm{ClN}_{5} \mathrm{O}_{3}$ (437.89): C, 60.34; H, 4.57; N, 16.00. Found: C, 60.22; $\mathrm{H}$, $4.72 ; \mathrm{N}, 15.66$

4-(3,7-Dimethyl-quinoxalin-2-yl)-6-(3,4,5-trimethoxy-phenyl)-pyrimidin-2-ylamine (7e). The derivative 7e was obtained as beige solid (13\%). IR (KBr) 3458-3360 (N-H), $1616(\mathrm{C}=\mathrm{N}), 1129(\mathrm{C}-\mathrm{O}-\mathrm{C}) ;{ }^{1} \mathrm{H}-\mathrm{NMR}\left(\mathrm{DMSO}-\mathrm{d}_{6}\right) \delta: 2.58$ $\left(\mathrm{s}, 3 \mathrm{H}, 7-\mathrm{CH}_{3}\right) ; 2.81\left(\mathrm{~s}, 3 \mathrm{H}, 3-\mathrm{CH}_{3}\right) ; 3.74\left(\mathrm{~s}, 3 \mathrm{H}, p-\mathrm{OCH}_{3}\right) ; 3.88\left(\mathrm{~s}, 6 \mathrm{H}, \mathrm{m}-\mathrm{OCH}_{3}\right) ; 6.96\left(\mathrm{~s}, 2 \mathrm{H}, \mathrm{NH}_{2}\right) ; 7.47(\mathrm{~s}, 2 \mathrm{H}$, $\left.\mathrm{H}_{2}{ }^{\prime}+\mathrm{H}_{6}{ }^{\prime}\right) ; 7.63(\mathrm{~s}, 1 \mathrm{H}$, ar- $\mathrm{CH}) ; 7.72-7.74\left(\mathrm{~d}, 1 \mathrm{H}, \mathrm{H}_{6}, J_{65}=8.57 \mathrm{~Hz}\right) ; 7.93\left(\mathrm{~s}, 1 \mathrm{H}, \mathrm{H}_{8}\right) ; 7.97-7.99\left(\mathrm{~d}, 1 \mathrm{H}, \mathrm{H}_{5}, J_{56}=8.58\right.$ $\mathrm{Hz}$ ); Analysis calculated for $\mathrm{C}_{23} \mathrm{H}_{23} \mathrm{~N}_{5} \mathrm{O}_{3}$ (417.47): C, 66.19; H, 5.52; N, 16.79. Found: C, 65.93; H, 5.61; N, 16.66.

General method for the synthesis of 2-methyl-3-[5-(3,4,5-trimethoxy-phenyl)-4,5-dihydro-1H-pyrazol-3-yl]quinoxaline derivatives (Series 8) and of 2-Methoxy-4-[5-(3-methyl-1,4-dioxy-quinoxalin-2-yl)-3,4-dihydro-2Hpyrazol-3-yl]-phenol derivatives (Series 10). $2 \mathrm{mmol}$ of hydrazine hydrate $98 \%$ were added to a solution of 0,5 mmol of the appropriate compound of series 6 in absolute ethanol. After 24 hours, the dissolvent was removed under reduced pressure and the solid obtained (compound of series 8 ) was filtered and washed with ethyl ether. In the same way, $2 \mathrm{mmol}$ of hydrazine hydrate $98 \%$ were added to a solution of $1 \mathrm{mmol}$ of the corresponding series 2 derivative and the reaction mixture was stirred for 24 hours. After removing the solvent under reduced pressure the residue was precipitated with ethyl acetate and the solid obtained (compounds of series 10) was filtered.

6-Methoxy-2-methyl-3-[5-(3,4,5-trimethoxy-phenyl)-4,5-dihydro-1H-pyrazol-3-yl]-quinoxaline (8c). The derivative 8c was obtained as yellow solid (74\%). IR (KBr) $3206(\mathrm{~N}-\mathrm{H}), 1592(\mathrm{C}=\mathrm{N}), 1127(\mathrm{C}-\mathrm{O}-\mathrm{C})$; ${ }^{1} \mathrm{H}-\mathrm{NMR}$ (DMSO-d $)_{6}$ ) $: 2.92\left(\mathrm{~s}, 3 \mathrm{H}, \mathrm{CH}_{3}\right) ; 3.10-3.17\left(\mathrm{dd}, 1 \mathrm{H}, \mathrm{H}_{\mathrm{A}}\right.$, upfield $\mathrm{H}$ of $\left.\mathrm{CH}_{2}, J_{\mathrm{AB}}=16.67 \mathrm{~Hz} ; J_{\mathrm{Ax}}=10.64 \mathrm{~Hz}\right) ; 3.65(\mathrm{~s}$, $\left.3 \mathrm{H}, p-\mathrm{OCH}_{3}\right) ; 3.68-3.74\left(\mathrm{dd}, 1 \mathrm{H}, \mathrm{H}_{\mathrm{B}}\right.$, downfield $\mathrm{H}$ of $\left.\mathrm{CH}_{2}, J_{\mathrm{BA}}=16.76 \mathrm{~Hz} ; J_{\mathrm{BX}}=11.30 \mathrm{~Hz}\right) ; 3.78\left(\mathrm{~s}, 6 \mathrm{H}, m-\mathrm{OCH}_{3}\right)$; $3.92\left(\mathrm{~s}, 3 \mathrm{H}, \mathrm{q}-\mathrm{OCH}_{3}\right) ; 4.89-4.95\left(\mathrm{ddd}, 1 \mathrm{H}, \mathrm{H}_{\mathrm{X}}, \mathrm{CH}, J_{\mathrm{XA}}=10.98 \mathrm{~Hz} ; J_{\mathrm{XB}}=11.06 \mathrm{~Hz} ; J_{\mathrm{X}-\mathrm{NH}}=2.10 \mathrm{~Hz}\right) ; 6.72(\mathrm{~s}, 2 \mathrm{H}$, $\left.\mathrm{H}_{2}{ }^{\prime}+\mathrm{H}_{6}{ }^{\prime}\right) ; 7.28-7.29\left(\mathrm{~d}, 1 \mathrm{H}, \mathrm{H}_{8}, J_{86}=2.76 \mathrm{~Hz}\right) ; 7.37-7.40\left(\mathrm{dd}, 1 \mathrm{H}, \mathrm{H}_{6}, J_{65}=9.12 \mathrm{~Hz} ; J_{68}=2.80 \mathrm{~Hz}\right) ; 7.85-7.87(\mathrm{~d}, 1 \mathrm{H}$, $\left.\mathrm{H}_{5}, J_{56}=9.11 \mathrm{~Hz}\right) ; 8.53\left(\mathrm{~d}, 1 \mathrm{H}, \mathrm{NH}, J_{\mathrm{NH}-\mathrm{X}}=2.13 \mathrm{~Hz}\right)$; Analysis calculated for $\mathrm{C}_{22} \mathrm{H}_{24} \mathrm{~N}_{4} \mathrm{O}_{4}(408.46): \mathrm{C}, 64.71 ; \mathrm{H}, 5.88$; N, 13.73. Found: C, 64.26; H, 5.72; N, 13.59 .

2,6,7-Trimethyl-3-[5-(3,4,5-trimethoxy-phenyl)-4,5-dihydro-1H-pyrazol-3-yl]-quinoxaline (8f). The derivative $8 \mathrm{f}$ was obtained as yellow solid (74\%). IR (KBr) $3205(\mathrm{~N}-\mathrm{H}), 1589(\mathrm{C}=\mathrm{N}), 1128(\mathrm{C}-\mathrm{O}-\mathrm{C}) ;{ }^{1} \mathrm{H}-\mathrm{NMR}\left(\mathrm{DMSO}-\mathrm{d}_{6}\right) \delta$ : 2.43-2.44 (2s, 6H, 6- $\left.\mathrm{CH}_{3}+7-\mathrm{CH}_{3}\right) ; 2.92\left(\mathrm{~s}, 3 \mathrm{H}, 3-\mathrm{CH}_{3}\right) ; 3.07-3.14\left(\mathrm{dd}, 1 \mathrm{H}, \mathrm{H}_{\mathrm{A}}\right.$, upfield $\mathrm{H}$ of $\mathrm{CH}_{2}, J_{\mathrm{AB}}=16.51 \mathrm{~Hz}$; $\left.J_{\mathrm{AX}}=11.05 \mathrm{~Hz}\right) ; 3.65\left(\mathrm{~s}, 3 \mathrm{H}, p-\mathrm{OCH}_{3}\right) ; 3.69-3.73\left(\mathrm{~m}, 1 \mathrm{H}, \mathrm{H}_{\mathrm{B}}\right.$, downfield $\mathrm{H}$ of $\left.\mathrm{CH}_{2}\right) ; 3.78\left(\mathrm{~s}, 6 \mathrm{H}, m-\mathrm{OCH}_{3}\right) ; 4.87-4.92$ $\left(\mathrm{t}, 1 \mathrm{H}, \mathrm{H}_{\mathrm{X}}, \mathrm{CH}, J_{\mathrm{XA}}=10.56 \mathrm{~Hz} ; J_{\mathrm{XB}}=10.56 \mathrm{~Hz}\right) ; 6.73\left(\mathrm{~s}, 2 \mathrm{H}, \mathrm{H}_{2}{ }^{\prime}+\mathrm{H}_{6}{ }^{\prime}\right) ; 7.71-7.73\left(2 \mathrm{~s}, 2 \mathrm{H}, \mathrm{H}_{5}+\mathrm{H}_{8}\right) ; 8.42(\mathrm{~s}, 1 \mathrm{H}, \mathrm{NH})$; Analysis calculated for $\mathrm{C}_{23} \mathrm{H}_{26} \mathrm{~N}_{4} \mathrm{O}_{3}$ (406.49): C, 67.98; H, 6.40; N, 13.79. Found: C, 67.65; H, 6.92; N, 13.63.

2-Methoxy-4-[5-(3-methyl-1,4-dioxy-quinoxalin-2-yl)-3,4-dihydro-2H-pyrazol-3-yl]-phenol (10a). The derivative 10a was obtained as yellow solid (35\%). IR (KBr) 3537-3317 (N-H, O-H), $1606(\mathrm{C}=\mathrm{N}), 1331(\mathrm{~N}-\mathrm{O}), 1273(\mathrm{C}-\mathrm{O})$; ${ }^{1} \mathrm{H}-\mathrm{NMR}\left(\mathrm{DMSO}_{6}\right) \delta$ : $2.60\left(\mathrm{~s}, 3 \mathrm{H}, \mathrm{CH}_{3}\right) ; 3.16-3.22\left(\mathrm{~m}, 1 \mathrm{H}, \mathrm{H}_{\mathrm{A}}\right.$, upfield $\mathrm{H}$ of $\left.\mathrm{CH}_{2}\right) ; 3.46-3.53\left(\mathrm{dd}, 1 \mathrm{H}, \mathrm{H}_{\mathrm{B}}\right.$, downfield $\mathrm{H}$ of $\left.\mathrm{CH}_{2}, J_{\mathrm{BA}}=16.23 \mathrm{~Hz} ; J_{\mathrm{BX}}=11.20 \mathrm{~Hz}\right) ; 3.79\left(\mathrm{~s}, 3 \mathrm{H}, \mathrm{OCH}_{3}\right) ; 4.88-4.93\left(\mathrm{~m}, 1 \mathrm{H}, \mathrm{H}_{\mathrm{X}}, \mathrm{CH}\right) ; 6.74-6.77$ (dd, $\left.1 \mathrm{H}, \mathrm{H}_{6}{ }^{\prime}, J_{6}{ }^{\prime}{ }^{\prime}=8.03 \mathrm{~Hz} ; J_{6}{ }^{\prime}{ }^{\prime}=1.42 \mathrm{~Hz}\right) ; 6.84-6.86\left(\mathrm{~m}, 1 \mathrm{H}, \mathrm{H}_{5}{ }^{\prime}\right) ; 7.06\left(\mathrm{~s}, 1 \mathrm{H}, \mathrm{H}_{2}{ }^{\prime}\right) ; 7.91-7.98\left(\mathrm{~m}, 2 \mathrm{H}, \mathrm{H}_{6}+\mathrm{H}_{7}\right) ; 8.11(\mathrm{~s}$, $1 \mathrm{H}, \mathrm{NH}) ; 8.46-8.51\left(\mathrm{~m}, 2 \mathrm{H}, \mathrm{H}_{5}+\mathrm{H}_{8}\right) ; 8.92(\mathrm{~s}, 1 \mathrm{H}, \mathrm{OH})$; Analysis calculated for $\mathrm{C}_{19} \mathrm{H}_{18} \mathrm{~N}_{4} \mathrm{O}_{4}$ (366.38): C, 62.30; $\mathrm{H}$, 4.92; N, 15.30. Found: C, 62.24; H, 5.18; N, 14.90 .

General method for the synthesis of 4-[3-(3-Methyl-quinoxalin-2-yl)-5-(3,4,5-trimethoxy-phenyl)-4,5-dihydropyrazol-1-yl]-benzenesulfonamide derivatives (Series 9). The corresponding compound of series 6 (0,55 mmol), 4hydrazinobenzene-1-sulfonamide hydrochloride $97 \%(0,55 \mathrm{mmol})$ and $10 \mathrm{~mL}$ of ethanol $99 \%$ were mixed in a microwave reaction flask. The program $(50 \mathrm{~W}, 5$ ') was carried out four times. The solid obtained was filtered and purified by flash column chromatography using ethyl acetate to yield compounds of series 9 .

4-[3-(3-Methyl-quinoxalin-2-yl)-5-(3,4,5-trimethoxy-phenyl)-4,5-dihydro-pyrazol-1-yl]-benzenesulfonamide (9a). The derivative 9a was obtained as yellow solid (14\%). IR (KBr) 3337-3248 (N-H), $1594(\mathrm{C}=\mathrm{N}), 1341$ (as 
$\mathrm{O}=\mathrm{S}=\mathrm{O}), 1158\left(\right.$ sym O=S=O), $1129(\mathrm{C}-\mathrm{O}-\mathrm{C}) ;{ }^{1} \mathrm{H}-\mathrm{NMR}\left(\right.$ acetone-d $\left._{6}\right) \delta: 3.20\left(\mathrm{~s}, 3 \mathrm{H}, \mathrm{CH}_{3}\right) ; 3.63-3.57\left(\mathrm{dd}, 1 \mathrm{H}, \mathrm{H}_{\mathrm{A}}\right.$, upfield $\mathrm{H}$ of $\left.\mathrm{CH}_{2}, J_{\mathrm{AB}}=18.10 \mathrm{~Hz} ; J_{\mathrm{AX}}=6.18 \mathrm{~Hz}\right) ; 3.71\left(\mathrm{~s}, 3 \mathrm{H}, p-\mathrm{OCH}_{3}\right) ; 3.78\left(\mathrm{~s}, 6 \mathrm{H}, m-\mathrm{OCH}_{3}\right) ; 4.22-4.30\left(\mathrm{dd}, 1 \mathrm{H}, \mathrm{H}_{\mathrm{B}}\right.$, downfield $\mathrm{H}$ of $\mathrm{CH}_{2}, J_{\mathrm{BA}}=18.11 \mathrm{~Hz} ; J_{\mathrm{BX}}=12.44 \mathrm{~Hz}$ ); 5.63-5.68 (dd, $\left.1 \mathrm{H}, \mathrm{H}_{\mathrm{X}}, \mathrm{CH}, J_{\mathrm{XA}}=6.15 \mathrm{~Hz} ; J_{\mathrm{XB}}=12.44 \mathrm{~Hz}\right) ; 6.36$ (s, 2H, NH $)_{2}$; $6.75\left(\mathrm{~s}, 2 \mathrm{H}, \mathrm{H}_{2}{ }^{\prime}+\mathrm{H}_{6}{ }^{\prime}\right) ; 7.31-7.34\left(\mathrm{~d}, 2 \mathrm{H}, \mathrm{H}_{\mathrm{C}}, J_{\mathrm{CD}}=8.96 \mathrm{~Hz}\right) ; 7.75-7.83\left(\mathrm{~d}, 2 \mathrm{H}, \mathrm{H}_{\mathrm{D}}, J_{\mathrm{DC}}=8.97 \mathrm{~Hz}\right) ; 7.79-$ $7.83\left(\mathrm{~m}, 2 \mathrm{H}, \mathrm{H}_{6}+\mathrm{H}_{7}\right)$; 7.99-8.03 (m, 2H, $\left.\mathrm{H}_{5}+\mathrm{H}_{8}\right)$; Analysis calculated for $\mathrm{C}_{27} \mathrm{H}_{27} \mathrm{~N}_{5} \mathrm{O}_{5} \mathrm{~S}$ (533.61): C, 60.79; $\mathrm{H}, 5.07$; N, 13.13. Found: C, 60.68; H, 5.22; N, 12.70.

4-[3-(7-Fluoro-3-methyl-quinoxalin-2-yl)-5-(3,4,5-trimethoxy-phenyl)-4,5-dihydro-pyrazol-1-yl]-

benzenesulfonamide (9b). The derivative 9b was obtained as yellow solid (29\%). IR (KBr) 3306-3243 (N-H), 1594 $(\mathrm{C}=\mathrm{N}), 1330($ as $\mathrm{O}=\mathrm{S}=\mathrm{O}), 1160($ sym $\mathrm{O}=\mathrm{S}=\mathrm{O}), 1130(\mathrm{C}-\mathrm{O}-\mathrm{C}) ;{ }^{1} \mathrm{H}-\mathrm{NMR}$ (acetone-d $\left.{ }_{6}\right) \delta: 3.19\left(\mathrm{~s}, 3 \mathrm{H}, \mathrm{CH}_{3}\right) ; 3.55-3.61$ $\left(\mathrm{dd}, 1 \mathrm{H}, \mathrm{H}_{\mathrm{A}}\right.$, upfield $\mathrm{H}$ of $\left.\mathrm{CH}_{2}, J_{\mathrm{AB}}=18.10 \mathrm{~Hz} ; J_{\mathrm{AX}}=6.20 \mathrm{~Hz}\right) ; 3.71\left(\mathrm{~s}, 3 \mathrm{H}, p-\mathrm{OCH}_{3}\right) ; 3.78\left(\mathrm{~s}, 6 \mathrm{H}, m-\mathrm{OCH}_{3}\right) ; 4.21-4.29$ $\left(\mathrm{dd}, 1 \mathrm{H}, \mathrm{H}_{\mathrm{B}}\right.$, downfield $\mathrm{H}$ of $\left.\mathrm{CH}_{2}, J_{\mathrm{BA}}=18.09 \mathrm{~Hz} ; J_{\mathrm{BX}}=12.47 \mathrm{~Hz}\right) ; 5.65-5.70\left(\mathrm{dd}, 1 \mathrm{H}, \mathrm{H}_{\mathrm{X}}, \mathrm{CH}, J_{\mathrm{XA}}=6.05 \mathrm{~Hz}\right.$; $\left.J_{\mathrm{XB}}=12.37 \mathrm{~Hz}\right) ; 6.36\left(\mathrm{~s}, 2 \mathrm{H}, \mathrm{NH}_{2}\right) ; 6.74\left(\mathrm{~s}, 2 \mathrm{H}, \mathrm{H}_{2}{ }^{\prime}+\mathrm{H}_{6}{ }^{\prime}\right) ; 7.32-7.35\left(\mathrm{~d}, 2 \mathrm{H}, \mathrm{H}_{\mathrm{C}}, J_{\mathrm{CD}}=8.96 \mathrm{~Hz}\right) ; 7.63-7.69(\mathrm{~m}, 2 \mathrm{H}$, $\left.\mathrm{H}_{6}+\mathrm{H}_{8}\right) ; 7.75-7.79\left(\mathrm{~d}, 2 \mathrm{H}, \mathrm{H}_{\mathrm{D}}, J_{\mathrm{DC}}=9.06 \mathrm{~Hz}\right) ; 8.06-8.10\left(\mathrm{~d}, 1 \mathrm{H}, \mathrm{H}_{5}, J_{56}=9.99 \mathrm{~Hz} ; J_{5 \mathrm{~F}}=5.80 \mathrm{~Hz}\right)$; Analysis calculated for $\mathrm{C}_{27} \mathrm{H}_{26} \mathrm{FN}_{5} \mathrm{O}_{5} \mathrm{~S}$ (551.60): C, 58.80; H, 4.72; N, 12.70. Found: C, 58.56; H, 4.76; N, 12.32 .

4-[3-(7-Methoxy-3-methyl-quinoxalin-2-yl)-5-(3,4,5-trimethoxy-phenyl)-4,5-dihydro-pyrazol-1-yl]benzenesulfonamide (9c). The derivative 9c was obtained as yellow solid (9.5\%). IR (KBr) 3313-3229 (N-H), 1593 $(\mathrm{C}=\mathrm{N}), 1326($ as $\mathrm{O}=\mathrm{S}=\mathrm{O}), 1155(\mathrm{sym} \mathrm{O}=\mathrm{S}=\mathrm{O}), 1122(\mathrm{C}-\mathrm{O}-\mathrm{C}) ;{ }^{1} \mathrm{H}-\mathrm{NMR}\left(\mathrm{DMSO}-\mathrm{d}_{6}\right) \delta: 3.10\left(\mathrm{~s}, 3 \mathrm{H}, \mathrm{CH}_{3}\right) ; 3.44-3.50$ $\left(\mathrm{dd}, 1 \mathrm{H}, \mathrm{H}_{\mathrm{A}}\right.$, upfield $\mathrm{H}$ of $\left.\mathrm{CH}_{2}, J_{\mathrm{AB}}=17.75 \mathrm{~Hz} ; J_{\mathrm{AX}}=5.51 \mathrm{~Hz}\right) ; 3.62\left(\mathrm{~s}, 3 \mathrm{H}, p-\mathrm{OCH}_{3}\right) ; 3.70\left(\mathrm{~s}, 6 \mathrm{H}, m-\mathrm{OCH}_{3}\right) ; 3.92(\mathrm{~s}$, $\left.3 \mathrm{H}, \mathrm{q}-\mathrm{OCH}_{3}\right)$; 4.10-4.18 (dd, $1 \mathrm{H}, \mathrm{H}_{\mathrm{B}}$, downfield $\mathrm{H}$ of $\left.\mathrm{CH}_{2}, J_{\mathrm{BA}}=18.17 \mathrm{~Hz} ; J_{\mathrm{BX}}=12.31 \mathrm{~Hz}\right) ; 5.61-5.65\left(\mathrm{dd}, 1 \mathrm{H}, \mathrm{H}_{\mathrm{X}}\right.$, $\left.\mathrm{CH}, J_{\mathrm{XA}}=5.56 \mathrm{~Hz} ; J_{\mathrm{XB}}=12.38 \mathrm{~Hz}\right) ; 6.90\left(\mathrm{~s}, 2 \mathrm{H}, \mathrm{H}_{2}{ }^{\prime}+\mathrm{H}_{6}{ }^{\prime}\right) ; 7.13\left(\mathrm{~s}, 2 \mathrm{H}, \mathrm{NH}_{2}\right) ; 7.22-7.24\left(\mathrm{~d}, 2 \mathrm{H}, \mathrm{H}_{\mathrm{C}}, J_{\mathrm{CD}}=8.55 \mathrm{~Hz}\right)$; 7.32-7.33 (d, $\left.1 \mathrm{H}, \mathrm{H}_{8}, J_{86}=2.47 \mathrm{~Hz}\right) ; 7.43-7.46\left(\mathrm{dd}, 1 \mathrm{H}, \mathrm{H}_{6}, J_{65}=8.83 \mathrm{~Hz} ; J_{68}=2.37 \mathrm{~Hz}\right) ; 7.68-7.71\left(\mathrm{~d}, 2 \mathrm{H}, \mathrm{H}_{\mathrm{D}}, J_{\mathrm{DC}}=\right.$ $8.70 \mathrm{~Hz}) ; 7.91-7.93\left(\mathrm{~d}, 1 \mathrm{H}, \mathrm{H}_{5}, J_{56}=9.07 \mathrm{~Hz}\right)$; Analysis calculated for $\mathrm{C}_{28} \mathrm{H}_{29} \mathrm{~N}_{5} \mathrm{O}_{6} \mathrm{~S}$ (563.64): C, 59.68; H, 5.15; N, 12.43. Found: C, 59.20; H, 4.96; N, 12.07.

4-[3-(7-Chloro-3-methyl-quinoxalin-2-yl)-5-(3,4,5-trimethoxy-phenyl)-4,5-dihydro-pyrazol-1-yl]-

benzenesulfonamide (9d). The derivative 9d was obtained as yellow solid (12\%). IR (KBr) 3302-3225 (N-H), 1594 $(\mathrm{C}=\mathrm{N}), 1341$ (as $\mathrm{O}=\mathrm{S}=\mathrm{O}), 1157(\operatorname{sym~O}=\mathrm{S}=\mathrm{O}), 1130(\mathrm{C}-\mathrm{O}-\mathrm{C}) ;{ }^{1} \mathrm{H}-\mathrm{NMR}\left(\mathrm{DMSO}-\mathrm{d}_{6}\right) \delta: 3.14\left(\mathrm{~s}, 3 \mathrm{H}, \mathrm{CH}_{3}\right) ; 3.40-3.46$ $\left(\mathrm{dd}, 1 \mathrm{H}, \mathrm{H}_{\mathrm{A}}\right.$, upfield $\mathrm{H}$ of $\left.\mathrm{CH}_{2}, J_{\mathrm{AB}}=17.80 \mathrm{~Hz} ; J_{\mathrm{AX}}=6.01 \mathrm{~Hz}\right) ; 3.62\left(\mathrm{~s}, 3 \mathrm{H}, p-\mathrm{OCH}_{3}\right) ; 3.70\left(\mathrm{~s}, 6 \mathrm{H}, m-\mathrm{OCH}_{3}\right) ; 4.09-4.17$ (dd, $1 \mathrm{H}, \mathrm{H}_{\mathrm{B}}$, downfield $\mathrm{H}$ of $\left.\mathrm{CH}_{2}, J_{\mathrm{BA}}=17.91 \mathrm{~Hz} ; J_{\mathrm{BX}}=12.41 \mathrm{~Hz}\right) ; 5.63-5.67\left(\mathrm{dd}, 1 \mathrm{H}, \mathrm{H}_{\mathrm{X}}, \mathrm{CH}, J_{\mathrm{XA}}=5.83 \mathrm{~Hz}\right.$; $\left.J_{\mathrm{XB}}=12.21 \mathrm{~Hz}\right) ; 6.61\left(\mathrm{~s}, 2 \mathrm{H}, \mathrm{H}_{2}{ }^{\prime}+\mathrm{H}_{6}{ }^{\prime}\right) ; 7.13\left(\mathrm{~s}, 2 \mathrm{H}, \mathrm{NH}_{2}\right) ; 7.24-7.26\left(\mathrm{~d}, 2 \mathrm{H}, \mathrm{H}_{\mathrm{C}}, J_{\mathrm{CD}}=8.91 \mathrm{~Hz}\right) ; 7.69-7.71\left(\mathrm{~d}, 2 \mathrm{H}, \mathrm{H}_{\mathrm{D}}\right.$, $\left.J_{\mathrm{DC}}=8.95 \mathrm{~Hz}\right) ; 7.81-7.84\left(\mathrm{dd}, 1 \mathrm{H}, \mathrm{H}_{6}, J_{65}=8.94 \mathrm{~Hz} ; J_{68}=2.28 \mathrm{~Hz}\right) ; 8.03-8.04\left(\mathrm{~d}, 1 \mathrm{H}, \mathrm{H}_{8}, J_{86}=2.45 \mathrm{~Hz}\right) ; 8.03-8.05(\mathrm{~d}$, $1 \mathrm{H}, \mathrm{H}_{5}, J_{56}=8.72 \mathrm{~Hz}$ ); Analysis calculated for $\mathrm{C}_{27} \mathrm{H}_{26} \mathrm{ClN}_{5} \mathrm{O}_{5} \mathrm{~S}$ (568.06): C, 57.09; H, 4.58; N, 12.33. Found: C, 56.68; $\mathrm{H}, 4.62 ; \mathrm{N}, 11.97$.

\section{Biological Experiments}

\section{Experiments in vitro}

In the in vitro assays each experiment was performed at least in triplicate and the standard deviation of absorbance was less than $10 \%$ of the mean.

Determination of the reducing activity of the stable radical 1,1-diphenyl-picrylhydrazyl (DPPH) ${ }^{33}$. To a solution of DPPH in absolute ethanol an equal volume of the compounds dissolved in DMSO was added. An ethanol solution was used as control. The concentrations of the solutions of the compounds were $0.05 \mathrm{mM}, 0.1$ and $0.2 \mathrm{mM}$. After 20 and $60 \mathrm{~min}$ at room temperature the absorbance was recorded at $517 \mathrm{~nm}$.

ABTS+• - Decolorization assay in ethanolic solution for antioxidant activity ${ }^{26}$. ABTS is dissolved in water to a 7 $\mathrm{mM}$ concentration. ABTS radical cation $\left(\mathrm{ABTS}^{+\bullet}\right.$ ) produced by reacting ABTS stock solution with $2.45 \mathrm{mM}$ potassium persulfate (final concentration) and allowing the mixture to stand in the dark at room temperature for $12-$ $16 \mathrm{~h}$ before use. For the present study, the $\mathrm{ABTS}^{+\bullet}$ solution was diluted with ethanol to an absorbance of 0.70 (60.02) at $734 \mathrm{~nm}$ and equilibrated at $30^{\circ} \mathrm{C}$. Stock solutions of the tested compounds in DMSO were diluted so that, after introduction of a $10-\mathrm{ml}$ aliquot of each dilution into the assay, they produced between $20 \%-80 \%$ inhibition of the blank absorbance. After addition of $1.0 \mathrm{ml}$ of diluted $\mathrm{ABTS}^{+\cdot}$ solution (A734nm) to $10 \mu 1$ of antioxidant compounds or Trolox standards (final concentration $0.1 \mathrm{mM}$ ) in ethanol the absorbance reading was taken at room temperature exactly 1 min after the initial mixing.

Non enzymatic assay of superoxide radicals-Measurement of superoxide radical scavenging activity ${ }^{33}$. The superoxide producing system was set up by mixing PMS, NADH and air-oxygen. The production of superoxide was estimated by the nitroblue tetrazolium method. The reaction mixture containing compounds, $3 \mu \mathrm{M}$ PMS, $78 \mu \mathrm{M}$ $\mathrm{NADH}$, and $25 \mu \mathrm{M}$ NBT in $19 \mu \mathrm{M}$ phosphate buffer $\mathrm{pH} 7.4$ was incubated for 2 min at room temperature and the absorption measured at $560 \mathrm{~nm}$ against a blank containing PMS. The tested compounds were preincubated for $2 \mathrm{~min}$ before adding NADH. 
Competition of the tested compounds with DMSO for hydroxyl radicals ${ }^{33}$. The hydroxyl radicals generated by the $\mathrm{Fe}^{3+}$ /ascorbic acid system, were detected according to Nash, by the determination of formaldehyde produced from the oxidation of DMSO. The reaction mixture contained EDTA $(0.1 \mathrm{mM}), \mathrm{Fe}^{3+}(167 \mu \mathrm{M})$, DMSO $(33 \mathrm{mM})$ in phosphate buffer $(50 \mathrm{mM}, \mathrm{pH} 7.4)$, the tested compounds (concentration $0.1 \mathrm{mM})$ and ascorbic acid (10 $\mathrm{mM})$. After 30 min of incubation $\left(37^{\circ} \mathrm{C}\right)$ the reaction was stopped with $\mathrm{CCl}_{3} \mathrm{COOH}(17 \% \mathrm{w} / \mathrm{v})$

Inhibition of linoleic acid lipid peroxidation ${ }^{31}$. Ten microliters of the $16 \mathrm{mM}$ linoleic acid dispersion was added to the UV cuvette containing $0.93 \mathrm{~mL}$ of $0.05 \mathrm{M}$ phosphate buffer, $\mathrm{pH} 7.4$ prethermostated at $37^{\circ} \mathrm{C}$. The oxidation reaction was initiated at $37{ }^{\circ} \mathrm{C}$ under air by the addition of $50 \mu \mathrm{L}$ of $40 \mathrm{mM}$ AAPH solution. Oxidation was carried out in the presence of aliquots in different concentrations. In the assay without antioxidant, lipid oxidation was measured in the presence of the same level of DMSO. The rate of oxidation at $37^{\circ} \mathrm{C}$ was monitored by recording the increase in absorption at $234 \mathrm{~nm}$ caused by conjugated diene hydroperoxides

Soybean lipoxygenase inhibition study in vitro. In vitro study was evaluated as reported previously. ${ }^{33}$ The tested compounds dissolved in DMSO were incubated at room temperature in different concentrations with sodium linoleate $(0.1 \mathrm{mM})$ and $0.2 \mathrm{ml}$ of enzyme solution $\left(1 / 9 \times 10^{-4} \mathrm{w} / \mathrm{v}\right.$ in saline $)$. The conversion of sodium linoleate to $13-$ hydroperoxylinoleic acid at $234 \mathrm{~nm}$ was recorded and compared with the appropriate standard inhibitor. $\mathrm{IC}_{50}$ values were determined.

\section{Experiments in vivo}

Inhibition of the carrageenin-induced edema. Edema was induced in the right hind paw of Fisher 344 rats (150$200 \mathrm{~g}$ ) by the intradermal injection of $0.1 \mathrm{ml} \%$ carrageenin in water. Both sexes were used. Females pregnant were excluded. Each group was composed of 6-15 animals. The animals, which have been bred in our laboratory, were housed under standard conditions and received a diet of commercial food pellets and water ad libitum during the maintenance but they were entirely fasted during the experiment period. Our studies were in accordance with recognised guidelines on animal experimentation.

The tested compounds $0.01 \mathrm{mmol} / \mathrm{kg}$ body weight, were suspended in water, with few drops of Tween 80 and ground in a mortar before use and were given intraperitoneally simultaneously with the carrageenin injection. The rats were euthanized $3.5 \mathrm{~h}$ after carrageenin injection. The difference between the weight of the injected and uninjected paws was calculated for each animal. The change in paw weight was compared with that in control animals (treated with water) and expressed as a percent inhibition of the edema CPE \% values. Indomethacin in 0.01 $\mathrm{mmol} / \mathrm{kg}$ presented $47 \%$ inhibition of the edema. Values CPE $\%$ are the mean from two different experiments with a standard error of the mean less than $10 \%$.

\section{Conclusions}

Several new quinoxaline and quinoxaline 1,4-di- $N$-oxide derivatives have been synthesized with the aim of studying their antioxidant and anti-inflammatory activities. We have optimized the synthesis of some of these derivatives by using microwave assisted methods that greatly improved reaction times and conversion ratios.

In terms of biological activity, the most interesting derivatives were those with the pyrazoline moiety (series $\mathbf{3 ,} 8$ and 10) and among them, those with $N$-oxide groups in the quinoxaline ring (series $\mathbf{3}$ and 10) exhibited significantly increased reducing activity compared to their reduced analogues (series 8). Compounds with an $\alpha, \beta$-unsaturated ketone system (1b, 2e, 4f, 5a, 5b, 5g and 6a-f) presented the best superoxide scavenging activities being the reduced derivatives (6a-f) the most interesting structures with interaction values between 79 and $100 \%$. This evidence led us to affirm that the olefinic moiety might play an important role in the activity of these compounds by trapping the superoxide radical. The derivatives are good hydroxyl radical scavengers. Compounds $\mathbf{6 b}$ and $\mathbf{6 e}$ were the best inhibitors of lipid peroxidation displaying $\mathrm{IC}_{50}$ values of $0.01 \mathrm{mM}$ and $<0.01 \mathrm{mM}$ respectively. In general, compounds that displayed good activities as inhibitors of lipid peroxidation also presented good values of inhibition of LOX. Compound $\mathbf{7 b}$ showed significant protection against carragenin induced paw edema.

\section{Acknowledgements}

The authors are grateful to Drs C. Hansch and A. Leo and to Biobyte Corp. for the free access to the C-QSAR program. Asunción Burguete was awarded a PhD fellowship supported by the "Gobierno de Navarra". Eleni Pontiki is grateful to the "Foundation for Education and European Culture" for financial support during PostDoc research.

\section{Notes and references}

1 Claesson, H. -E.; Dahlen, S. -E. J. Intern. Med. 1999, 245, 205-227.

2 Dwyer, J. H.; Allayee, H.; Dwyer, K. M.; Fan, J.; Wu, H.; Mar, R.; Lusis, A. J.; Mehrabian, M. N. Engl. J. Med. $2004,350,29-37$.

3 Hennig, R.; Ding, X.; Tong, W; Schneider, M. B.; Standop, J.; Friess, H.; Büchler, M. W.; Pour, P. M.; Adrian, T. E. Am. J. Pathol. 2002, 161, 421-428.

4 Yoshimura, R.; Matsuyama, M.; Tsuchida, K.; Kawahito, Y.; Sano, H.; Nakatani, T. J. Urol. 2003, 170, $1994-1999$.

5 Jiang, W. G.; Douglas-Jones, A.; Mansel, R. E. Prostaglandins Leukot. Essent. Fatty Acids. 2003, 69, $275-281$. 
6 Franco, R.; Schoneveld, O.; Georgakilas, A. G.; Panayiotidis, M. I. Cancer Lett. 2008, 266, 6-11.

7 Dhalla, N. S.; Temsah, R. M.; Netticadan, T. J. Hypertens. 2000, 18, 655-673.

8 Chen, L.; Liu, L.; Yin, J.; Luo, Y.; Huang, S. Int. J. Biochem. Cell Biol. 2009, 41, 1284-1295.

9 Halliwell, B.; Hoult, J. R.; Blake, D. R. FASEB J. 1988, 2, 2867-2873.

10 Weiss, S. J. N. Engl. J. Med. 1989, 320, 365-376.

11 Udassin, R.; Ariel, I.; Haskel, Y.; Kitrossky, N.; Chevion, M. Free Radic. Biol. Med. 1991, 10, 1-6.

12 Tsujimoto, Y.; Saitoh, K.; Kashima, M.; Shiozawa, A.; Kozuka, M.; Hashizume, H.; Kimura, K.; Yamazaki, M.; Fujii, A. Gen. Pharmacol. 1998, 31, 405-408.

13 Maharaj, H.; Maharaj, D. S.; Daya, S. Metab. Brain Dis. 2006, 21, 189-199.

14 Facino, R. M.; Carini, M.; Aldini, G.; Saibene, L.; Morelli, R. Arzneim. Forsch. Drug Res. 1995, 45-2, $1102-1109$.

15 Vicente, E.; Villar, R.; Burguete, A.; Solano, B.; Perez-Silanes, S.; Aldana, I.; Maddry, J. A.; Lenaerts, A. J.; Franzblau, S. G.; Cho, S.; Monge, A.; Goldman, R. C. Antimicrob. Agents Chemother. 2008, 52, 3321-3326.

16 Zarranz, B.; Jaso, A.; Aldana, I.; Monge, A. Bioorg. Med. Chem. 2004, 12, 3711-3721.

17 Burguete, A.; Estevez, Y.; Castillo, D.; Gonzalez, G.; Villar, R.; Solano, B.; Vicente, E.; Pérez, S.; Aldana, I.; Monge, A.; Sauvain, M.; Deharo, E. Mem. Inst. Oswaldo Cruz. 2008, 103, 778-780.

18 Burguete, A.; Pontiki, E.; Hadjipavlou-Litina, D.; Villar, R.; Vicente, E.; Solano, B.; Ancizu, S.; Pérez-Silanes, S.; Aldana, I.; Monge, A. Bioorg. Med. Chem. Lett. 2007, 17, 6439-6443.

19 Monge, A.; Palop, J. A.; De Cerain, A. L.; Senador, V.; Martinez Crespo, F. J.; Sainz, Y.; Narro, S.; Garcia, E.; Demiguel, C.; Gonzalez, M.; Hamilton, E.; Barker, A. J.; Clarke, E. D.; Greenhow, D. T. J. Med. Chem. 1995, 38, $1786-1792$.

20 Monge, A.; Gil, M. J.; Gastelurrutia, M. A.; Pascual, M. An. Real Acad. Farm. 1982, 48, 533-542.

21 Johnston, J. D. U.S. Patent 3371090, 1968.

22 Haddadin, M. J.; Issidorides, C. H. Heterocycles. 1976, 4, 767-816.

23 Solano, B.; Junnotula, V.; Marin, A.; Villar, R.; Burguete, A.; Vicente, E.; Perez-Silanes, S.; Aldana, I.; Monge, A.; Dutta, S.; Sarkar, U.; Gates, K. S. J. Med. Chem. 2007, 50, 5485-5492.

24 Jaso, A.; Zarranz, B.; Aldana, I.; Monge, A. Eur. J. Med. Chem. 2003, 38, 791-800.

25 Reddy, M. V.; Billa, V. K.; Pallela, V. R.; Mallireddigari, M. R.; Boominathan, R.; Gabriel, J. L.; Reddy, E. P. Bioorg. Med. Chem. 2008, 16, 3907-3916.

26 Re, R.; Pellegrini, N.; Proteggente, A.; Pannala, A.; Yang, M.; Rice-Evans, C. Free Radic. Biol. Med. 1999, 26, $1231-1237$.

27 Valko, M.; Leibfritz, D.; Moncol, J.; Cronin, M. T. D.; Mazur, M.; Telser, J. Int. J. Biochem. Cell Biol. $2007,39,44-84$.

28 Ames, B. N.; Shigenaga, M. K.; Hagen, T. M. Proc. Natl. Acad. Sci. USA. 1993, 90, 7915-7922.

29 Pontiki, E.; Hadjipavlou-Litina, D. Med. Chem. 2006, 2, 251-264.

30 Halliwell, B.; Gutteridge, J. M. C. Biochem. J. 1984, 219, 1-14.

31 Liegeois, C.; Lermusieau, G.; Collin, S. J. Agric. Food Chem. 2000, 48, 1129-1134.

32 Taraporewala, I. B.; Kauffman, J. M. J. Pharm. Sci. 1990, 79, 173-178.

33 Pontiki, E.; Hadjipavlou-Litina, D. Bioorg. Med. Chem. 2007, 15, 5819-5827.

34 Non-steroidal Anti-inflammatory Drugs; Winter, C. A. and In: Garattini, S. and Dukes, M.N.G., Eds.; Excepta Medica.: Amsterdam, 1965.

35 Kuroda, T.; Suzuki, F.; Tamura, T.; Ohmori, K.; Hosoe, H. J. Med. Chem. 1992, 35, 1130-1136.

36 Kim, H. K.; Miller, L. F.; Bambury, R. E.; Ritter, H. W. J. Med. Chem. 1977, 20, 557-560.

37 www. biobyte.com, Biobyte Corp., C-QSAR Database 201 West $4^{\text {th }}$ Str., Suite 204, Claremont CA, California 91711 , USA. 